\title{
Transcriptome profiling of anthocyanin biosynthesis in the peel of 'Granny Smith' apples (Malus domestica) after bag removal
}

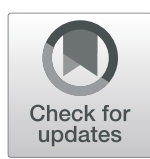

Changqing Ma ${ }^{1,2}$, Bowen Liang ${ }^{1}$, Bo Chang ${ }^{1,2}$, Jiuying Yan ${ }^{1,2}$, Li Liu ${ }^{1,2}$, Ying Wang ${ }^{1,2}$, Yazhou Yang ${ }^{1,2}$ and Zhengyang Zhao ${ }^{1,2^{*}}$ (D)

\begin{abstract}
Background: Bagging is commonly used to enhance red pigmentation and thereby improve fruit quality of apples (Malus domestica). The green-skinned apple cultivar 'Granny Smith' develops red pigmentation after bagging removal, but the signal transduction pathways mediating light-induced anthocyanin accumulation in apple peel are yet to be defined. The aim of this study was to identify the mechanisms underpinning red pigmentation in 'Granny Smith' after bag removal based on transcriptome sequencing.

Results: The anthocyanin content in apple peel increased considerably after bag removal, while only trace amounts of anthocyanins were present in the peel of unbagged and bagged fruits. RNA sequencing identified 18,152 differentially expressed genes (DEGs) among unbagged, bagged, and bag-removed fruits at 0, 4, and 10 days after bag removal. The DEGs were implicated in light signal perception and transduction, plant hormone signal transduction, and antioxidant systems. Weighted gene co-expression network analysis of DEGs generated a module of 23 genes highly correlated with anthocyanin content. The deletion of -2026 to $-1870 \mathrm{bp}$ and -1062 to -964 bp regions of the MdMYB1 (LOC103444202) promoter induced a significant decrease in glucuronidase activity and anthocyanin accumulation in apple peel.

Conclusions: Bagging treatment can induce red pigmentation in 'Granny Smith' via altering the expression patterns of genes involved in crucial signal transduction and biochemical metabolic pathways. The -2026 to $1870 \mathrm{bp}$ and -1062 to $-964 \mathrm{bp}$ regions of the MdMYB1 promoter are essential for MdMYB1-mediated regulation of anthocyanin accumulation in the 'Granny Smith' apple cultivar. The findings presented here provide insight into the mechanisms of coloration in the peel of 'Granny Smith' and other non-red apple cultivars.
\end{abstract}

Keywords: Anthocyanin content, Apple bagging, Differentially expressed genes, Granny Smith cultivar, RNA sequencing, MdMYB1

\section{Background}

Apple (Malus domestica) is a highly valued and widely cultivated fruit crop around the world. From a consumer's perspective, apples with red peel are preferred to other colors in terms of their appearance and nutritional value [1]. The red color of apple peel is due to the presence of anthocyanins [2], a natural source of antioxidants with high nutritional value and potential health benefits [3, 4].

\footnotetext{
*Correspondence: zhaozy@nwsuaf.edu.cn; applebreed@126.com ${ }^{1}$ State Key Laboratory of Crop Stress Biology for Arid Areas, College of Horticulture, Northwest A \& F University, Yangling 712100, Shaanxi, China ${ }^{2}$ Shaanxi Research Center of Apple Engineering and Technology, Yangling

712100, Shaanxi, China
}

Anthocyanins are biosynthesized through the flavonoid pathway that involves many structural and regulatory genes $[5,6]$. The structural genes associated with anthocyanin biosynthesis in apple have been characterized, including phenylalanine ammonia lyase (PAL), chalcone synthase (CHS), 4-coumarate coenzyme A ligase (4CL), chalcone isomerase (CHI), dihydroflavonol 4-reductase (DFR), flavanone 3-hydroxylase (F3H), anthocyanidin synthase/leucoanthocyanidin dioxygenase (ANS), and UDP-glucose:flavonoid 3-O-glucosyltransferase (UFGT) [7, 8]. All these structural genes are regulated by a conserved MYB-bHLH-WD40/ WDR (MBW) regulatory complex [9]. Transcriptional levels

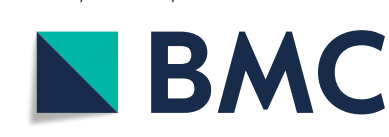

(c) The Author(s). 2019 Open Access This article is distributed under the terms of the Creative Commons Attribution 4.0 International License (http://creativecommons.org/licenses/by/4.0/), which permits unrestricted use, distribution, and reproduction in any medium, provided you give appropriate credit to the original author(s) and the source, provide a link to the Creative Commons license, and indicate if changes were made. The Creative Commons Public Domain Dedication waiver (http://creativecommons.org/publicdomain/zero/1.0/) applies to the data made available in this article, unless otherwise stated. 
of $M Y B$ are correlated with anthocyanin biosynthesis in red-skinned apple cultivars, and in red regions of apple peel [10]. By regulating the levels of structural gene transcripts, methylation of the apple $M d M Y B 1$ promoter affects the formation of red pigments in apple peel [11, 12]. A single-nucleotide polymorphism in the $M d M Y B 1$ promoter causes abnormalities of anthocyanin biosynthesis [13].

Anthocyanin accumulation in plant can be regulated by light and plant hormones [14, 15]. Under light conditions, plant photoreceptors perceive and transduce light signals to regulate anthocyanin biosynthesis [16]. The classical photoreceptors include phytochromes (PHYs), cryptochromes (CRYs), and phototropins (PHOTs) that perceive light signals ranging from ultraviolet (UV)-A to far-red [17]. Exposure to UV-B irradiation promotes anthocyanin biosynthesis, resulting in binding and activation of promoter regions of $M d M Y B$ genes in apple peel [18]. UVR8 is a UV-B photoreceptor that plays vital roles in UV-B induction of flavonoid biosynthesis and plant defense against UV-B [19-21]. Downstream of the photoreceptors, CONSTITUTIVE PHOTOMORPHOGENIC 1 (COP1) [17], LONG HYPOCOTYL 5 (HY5) [16], suppressor of phyA (SPA) [22], DE-ETIOLATED (DET) [23], and PHYTOCHROME KINASE SUBSTRATE 1 (PKS1) [24] also participate in light-induced plant development. In addition, plant hormones regulate the expression of anthocyanin biosynthetic genes in a light-dependent manner [25]. For example, jasmonic acid modulates anthocyanin biosynthetic genes expression toward the end of the pathway, while cytokinins induce the expression of both early and late anthocyanin biosynthetic genes in Arabidopsis [26].

Anthocyanins play a photoprotective role under certain stress conditions, such as high light exposure [27]. The photoprotective function of anthocyanins may be mainly attributed to direct scavenging of reactive oxygen species (ROS) [28, 29]. Various enzymatic antioxidant systems are present in cells, including superoxide dismutase (SOD), catalase (CAT), peroxidase (POD), ascorbate peroxidase (APX), glutathione reductase (GR), monodehydroascorbate reductase (MDHAR), glutathione peroxidase (GPX), and glutathione-S-transferase (GST), that work in concert to prevent uncontrolled oxidation [30-32]. In apples, the relationship between anthocyanin biosynthesis and antioxidant systems is dependent on light conditions. Unlike the non-red cultivar 'Golden Delicious', antioxidant systems in the red cultivar 'Red Delicious' are initially upregulated by anthocyanins during sunlight exposure, but at higher anthocyanin concentrations downregulation can occur [33]. However, whether these enzymatic antioxidant systems are also involved in red pigmentation in non-red apple cultivars after bag removal have not been well documented.
The 'Granny Smith' is a green-skinned apple cultivar, but fruits can turn cardinal red after bag removal, as practiced in the Loess Plateau region of China to improve apple quality [34]. Recently, several studies have attempted to explain this coloration phenomenon in 'Granny Smith'. The major anthocyanin pigment present in 'Granny Smith' peel is cyanidin 3-galactoside [35]. Transcriptional levels of $M d M Y B 1$ are correlated with anthocyanin biosynthesis in the peel of 'Granny Smith' fruits [36], which display reduced methylation in the 2026 to $-1870 \mathrm{bp}$ and -1062 to $-964 \mathrm{bp}$ regions of the $M d M Y B 1$ promoter after bag removal [11]. Expression of the structural genes $M d F 3 H, M d D F R, M d A N S$, and $M d U F G T$ that are involved in anthocyanin biosynthesis is considerably increased in 'Granny Smith' apple peel after bag removal [36]. However, the signal transduction pathways mediating light-induced anthocyanin accumulation in 'Granny Smith' after bag removal are yet to be defined.

The objective of this study was to investigate the molecular mechanisms and the metabolic pathways involved in the regulation of red pigmentation induced by bagging treatment, and to identify the key genes involved in regulating anthocyanin biosynthesis in the peel of 'Granny Smith' apples after bag removal. Based on transcriptome sequencing of 'Granny Smith' fruit peel after bag removal, we identified differentially expressed genes (DEGs) involved in light responses, hormone signal transduction pathways, and enzymatic antioxidant systems. By constructing co-expression networks, we revealed the differential regulation of genes involved in anthocyanin accumulation. The findings help to further clarify the regulatory mechanisms of bagging treatment-induced red pigmentation in 'Granny Smith' apples and possibly other non-red fruits.

\section{Results}

Changes in fruit pigmentation patterns in apple peel

By visual inspection, fruits gradually turned red after bag removal, while unbagged and bagged fruits remained green and white, respectively (Fig. 1a). The red pigmentation of fruits removed from bags [160 days after full bloom (DAFB)] was associated with an elevation (0 to $0.39 \mathrm{mg} \mathrm{g}^{-1}$ ) in the anthocyanin content of fruit peel samples from 0 to 10 days after bag removal (DABR), while only trace amounts of anthocyanins were observed in unbagged $\left(\sim 0.01 \mathrm{mg} \mathrm{g}^{-1}\right)$ and bagged $\left(0 \mathrm{mg} \mathrm{g}^{-1}\right)$ fruits (Fig. 1b). Additionally, the chlorophyll content in the peel of bagged fruits and bag-removed fruits remained at $\sim 0.04 \mathrm{mg} \mathrm{g}^{-1}$, which was significantly lower than in unbagged fruits; no significant difference was observed in chlorophyll content between bagged and bag-removed fruits (Fig. 1c). These findings indicate that the red pigmentation can be mainly attributed to the accumulation of anthocyanins in the peel of 'Granny Smith' apples. 


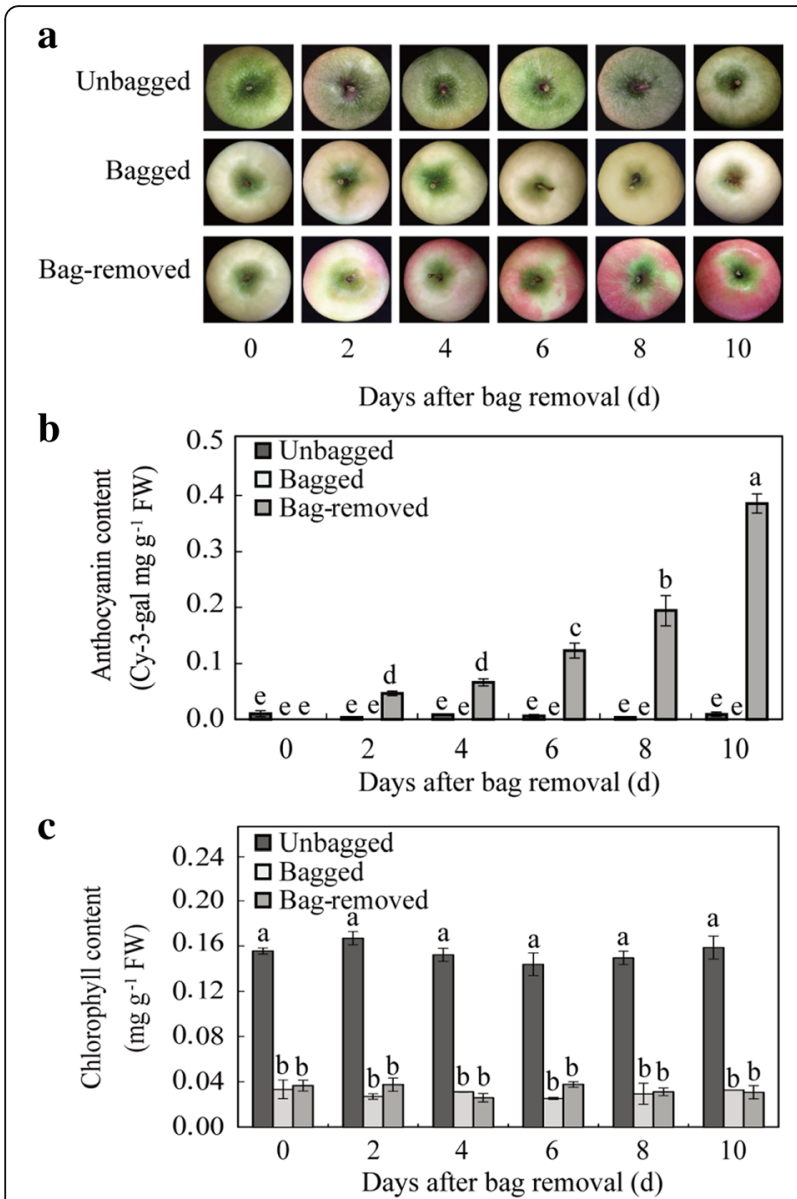

Fig. 1 Changes in peel color and pigmentation in the 'Granny Smith' apple cultivar after bag removal. a Color development in apple peel. b Changes in cyanidin3-galactoside and (c) chlorophyll content in fruit peel. Error bars indicate standard deviation of biological replicates. Different lowercase letters indicate significant differences among bag-removed, unbagged, and bagged fruits by Tukey's multiple range tests $(p<0.05)$

\section{Characterization of unbagged, bagged and bag-removed apple transcriptomes}

To gain global insight into the molecular mechanisms underpinning the different phenotypes, we analyzed fruit peel samples of unbagged (U), bagged (B), and bag-removed (R) groups at 0 (U0/B0/R0), 4 (U4/B4/R4), and 10 (U10/ B10/R10) DABR by RNA sequencing (RNA-seq). After stringent quality filtering, the number of clean reads per library ranged from 24.40 to 32.42 million (M), and the rate of reads mapped to the genome was 62.56 to $73.35 \%$, with a uniquely mapped rate of 56.44 to $65.29 \%$ (Additional file 1 : Tables S1 and S2). All biological replicates showed a strong correlation $\left(\mathrm{R}^{2} \geq 0.99\right.$; Additional file 1: Table S3).

\section{Identification of DEGs}

DEGs were identified among samples of each treatment group at 0,4 , and 10 DABR. More DEGs were found between R0 vs. R4 than R4 vs. R10, and similar results were also obtained for the $\mathrm{B}$ and $\mathrm{U}$ groups (Fig. $2 \mathrm{a}$ and $\mathrm{c}$ ). This result indicates that the stage between 0 and 4 DABR may be the critical period affecting fruit pigmentation and development in apple peel. DEGs between and among different treatment groups were also identified. The number of DEGs between U vs. B (or B vs. R) slightly increased following bag removal, while the reverse was true for that between $U$ vs. R. The identified DEGs were selected for further analysis (Fig. 2b and d).

\section{Changes in gene expression profiles in different treatment groups}

To gain further insight into related biological processes, transcripts could be divided into eight clusters in each treatment group (Additional file 2: Figure S1), representing distinct expression patterns. Then, we performed Kyoto Encyclopedia of Genes and Genomes (KEGG) pathway enrichment analysis to provide a global perspective of the biological pathways enriched in each cluster of similarly regulated transcripts (Fig. 3). Cluster 7 in both the $R$ and $U$ groups contained genes positively modulated throughout the entire time course, many of which were involved in flavonoid biosynthesis (Fig. 3a and b). Cluster 6 in the R group contained genes upregulated at 0 DABR, including those involved in photosynthesis, porphyrin and chlorophyll metabolism, carotenoid biosynthesis, carbon metabolism, and carbon fixation in photosynthetic organisms (Fig. 3a). Reversely, the above-mentioned genes were significantly downregulated from 0 to 4 DABR in the $B$ group (Fig. 3c). In addition, genes involved in the 'peroxisome' category were significantly downregulated from 0 to 4 DABR in the $U$ and B groups (Fig. $3 \mathrm{~b}$ and $\mathrm{c}$ ). These results indicate these biological pathways have potentially important effect on apple color.

\section{Expression of genes involved in light signal perception and transduction}

Following bag removal, apple responses to light were mediated by different classes of photoreceptors (Fig. 4a). Both PHYA and PHYC were expressed at low levels in the $\mathrm{R}$ group, with the highest levels found in $\mathrm{B} 0$ and U10, respectively. Transcription of two PHYBs also remained low in the R group; LOC103423949 expression peaked in B0, while LOC103431933 expression was highest in U10 and B0. Additionally, expression of two PHOT1s (LOC103412999 and LOC103422828) was low in the R group, with the highest levels found in B10. By contrast, two PHYEs displayed high expression in U10, and levels gradually increased in $\mathrm{R}$ group. Among CRYs, expression of LOC103425741 increased rapidly in the $\mathrm{R}$ group and peaked in R4, whereas the other four members exhibited low expression in this group. Among PHOT2s, expression of LOC108169229 increased in the $\mathrm{R}$ group and peaked in R10, whereas LOC103428062 


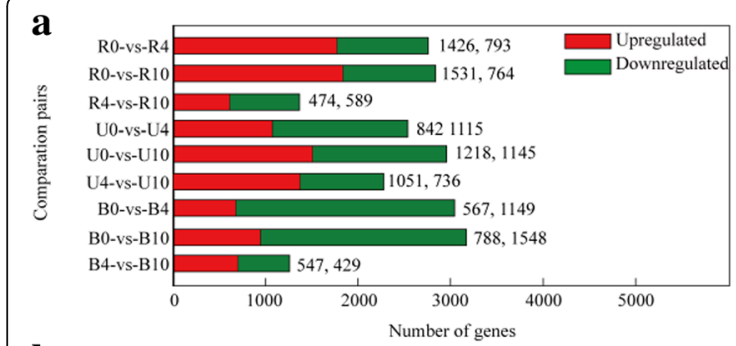

b

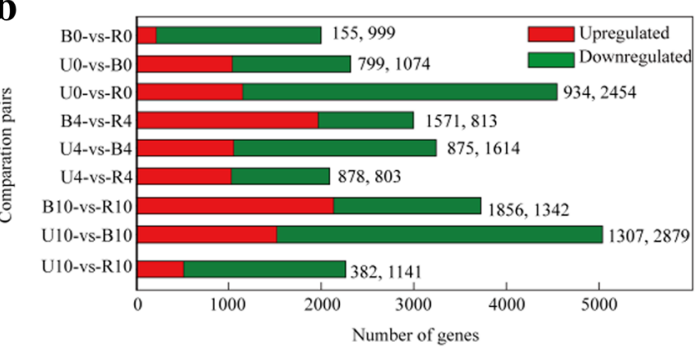

c

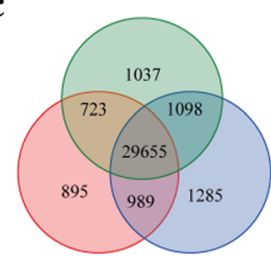

oU0 oU4 oU10

d

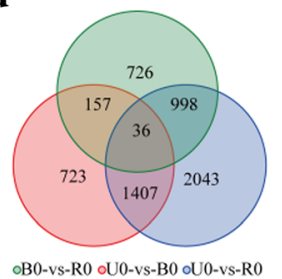

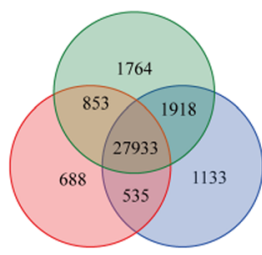

$\circ \mathrm{B} 0 \quad \mathrm{oB} 4 \quad \mathrm{oB} 10$

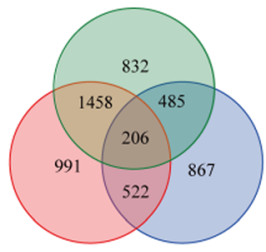

oB4-vs-R4 oU4-vs-B4 oU4-vs-R4 oB 10-vs-R 10 ○U10-vs-B 10 oU 10 -vs-R 10

Fig. 2 Differentially expressed genes (DEG) identified by RNA-seq analysis in the peel of bag-removed (R), unbagged (U), and bagged (B) fruits at varying developmental stages. a Number of DEGs between samples in each treatment groups at 0, 4, and 10 days after bag removal (DABR) (b) Number of DEGs between U vs. R, U vs. B, and B vs. R groups at 0, 4, and 10 DABR (c) Venn diagram representation of DEGs from pairwise comparisons. $\mathbf{d}$ Venn diagram representation of total genes from 0 to $10 \mathrm{DABR}$ in $\mathrm{R}, \mathrm{U}$, and $\mathrm{B}$ groups. FDR $<0.05$ and $\left|\log _{2} \mathrm{FC}\right|>1$ were used as cut-off criteria for significance. 0-, 0 DABR; - 4, 4 DABR; and - 10, 10 DABR

and LOC103442690 exhibited low expression in this group. Moreover, eight genes encoding UVR8 proteins were identified; expression of LOC103401151, LOC103410004, and LOC103441159 decreased in the R group, while levels of the other five were increased to varying degrees.

Light signal transduction genes were also among the DEGs identified in apple peel (Fig. 4b). Low expression of COP1s was found in the $\mathrm{R}$ group compared with the $\mathrm{U}$ and B groups. Expression of COP10 (LOC103415035) decreased in both $U$ and $\mathrm{R}$ groups, with peak levels found in B4. SPAs were expressed at low levels in the $\mathrm{R}$ group, while expression significantly increased in the $\mathrm{U}$ group. DET (LOC103409319) expression levels were highest in U10 and R4. Expression of two PKS1s gradually increased in the $\mathrm{R}$ group; LOC103416646 was highly expressed in U0 and U10, while LOC103425574 reached its peak levels in U10 and B0. In addition, PKS3 exhibited low expression in the $U$ group, but levels gradually increased in the other groups and peaked in B4 and R4. These results indicate that the light signal perception and transduction patterns in apple peel could be dynamically altered by bagging treatment.

Expression of genes associated with phytohormone signaling To determine the functions of plant hormones in bagginginduced pigmentation, we assessed the expression patterns of hormone signaling-related genes encoding receptors and response factors. The heat map shows that the functions of genes involved in plant hormone signaling differed across the three treatment groups (Fig. 5).
In the ethylene transduction pathway, the transcriptional level of serine/threonine-protein kinase CTR1 (CTR1, LOC103423189) was low in the U group, while this gene was upregulated in B4 and R4. Meanwhile, expression of the ethylene receptor (ETR) and ethylene-insensitive protein (EIN) genes was low in the R group. In contrast, expression levels of genes encoding auxin influx carrier/auxin-responsive protein IAA (AUX/IAA), auxin response factor (ARF), auxin responsive GH3 gene family (GH3), and small auxin up RNA (SAUR) were significantly upregulated in the $\mathrm{R}$ group.

In the abscisic acid transduction pathway, genes encoding the abscisic acid receptor (PYR/PYL) and protein phosphatase 2C (PP2C) were highly expressed in the $\mathrm{R}$ group. Reversely, expression of serine/threonine protein kinase SRK2n (SnRK2) genes was low in the $\mathrm{R}$ group, with high levels found in U0 and B0.

In the cytokinin pathway, expression of histidine-containing phosphotransfer protein (AHP) genes peaked in R0, while expression of two-component response regulator ARR-A and ARR-B families peaked in R4. Moreover, expression of the jasmonic acid-amino synthetase (JAR1) gene was high in R0 and R10, while low transcriptional levels were found in the $U$ and $B$ groups. Similarly, expression of the transcription factor $M Y C 2$ peaked in R10. In contrast, expression of four jasmonate ZIM (JAZ) domain-containing genes was low in the $\mathrm{R}$ group, and levels peaked in U4. These results indicate that the genes involved in phytohormone signaling follow distinct expression patterns in apple peel during the bagging treatment. 

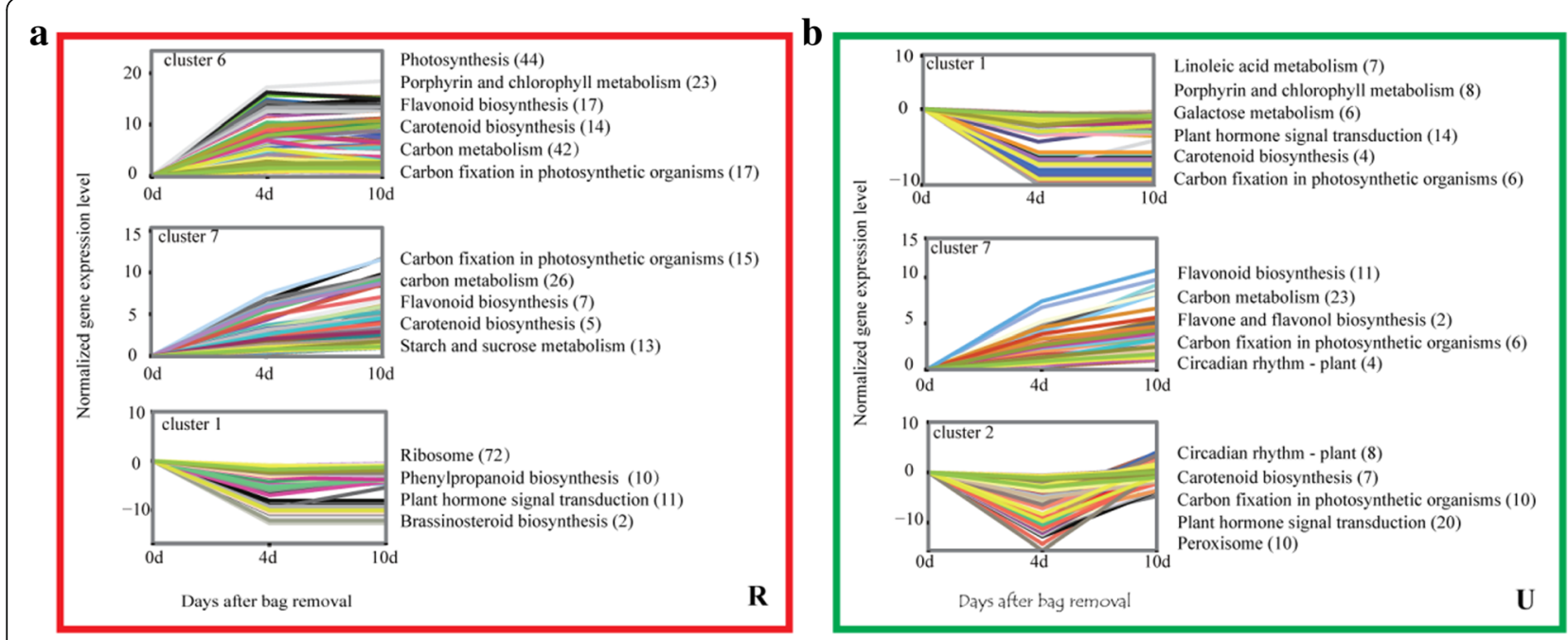

c

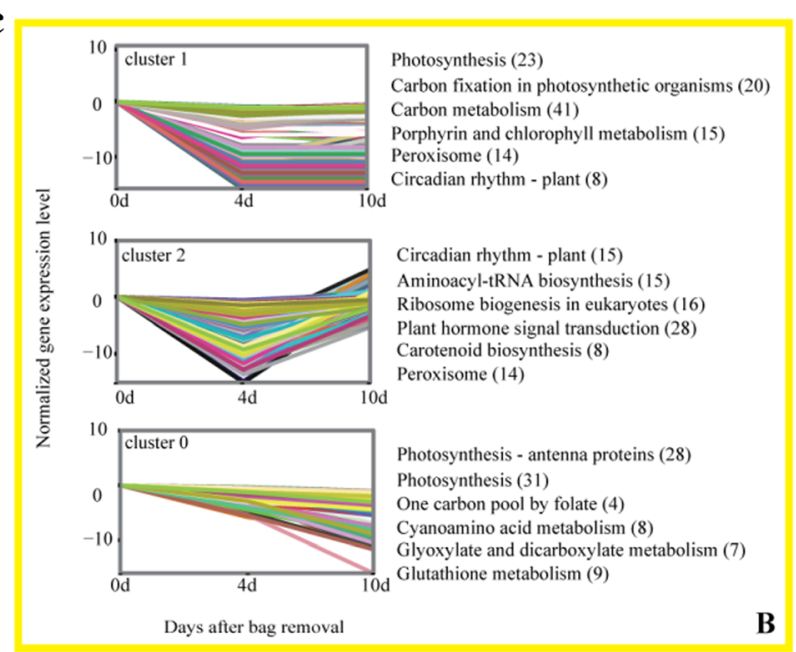

Fig. 3 Cluster analysis and Kyoto Encyclopedia of Genes and Genomes (KEGG) pathway enrichment analysis of differentially expressed transcripts exhibiting significant expression profile changes, and KEGG pathway enrichment analysis. Enriched KEGG pathways are listed to the right of each cluster. (a), (b) and (c) Changes in gene expression profiles and KEGG pathways in bag-removed, unbagged, and bagged fruits, respectively

Expression of genes related to antioxidant enzyme systems To investigate the effect of light exposure on antioxidant enzyme systems, we analyzed the expression of some important antioxidant genes across the three treatment groups. SODs, CATs, POXs, APXs, GRs, MDHARs, GPXs, and GSTs were upregulated during the pigmentation of 'Granny Smith' fruits (Fig. 6). Notably, global gene expression patterns were higher in $\mathrm{R}$ than in the other two groups, except for one CAT (LOC103429547), two GRs (LOC103443500 and LOC103456002), and two MDHARs (LOC103404333 and LOC103411764). Following bag removal, expression of most antioxidant enzyme-encoding genes peaked in R4. However, two SODs, six PODs, and 12 GSTs exhibited relatively high expression in R0, followed by a decrease thereafter. These findings indicate that light exposure could induce transient and significant regulation of transcripts that encode antioxidant enzymes in apple peel. Moreover, some genes peaked in R10, including two SODs, 10 PODs, two GPXs, and seven GSTs, which exhibited similar trends as the anthocyanin content in apple peel of the R group (Fig. 1b).

\section{Identification of co-expression network modules associated with anthocyanin accumulation}

A weighted gene co-expression network analysis (WGCNA) was performed on the 18,152 non-redundant DEGs identified above, leading to the identification of 16 modules (Fig. 7a and b). Analysis of module-trait relationships revealed that the 'darkolivegreen 4' module was highly correlated with anthocyanin content $(r=0.48, p=0.01)$ in the 27 samples (Fig. 4b). Further module-trait relationship analysis, using the expression level of MYB1 (LOC103444202) and UFGT (LOC103417897) as trait data, revealed that their expression patterns were indeed highly correlated with this module for 


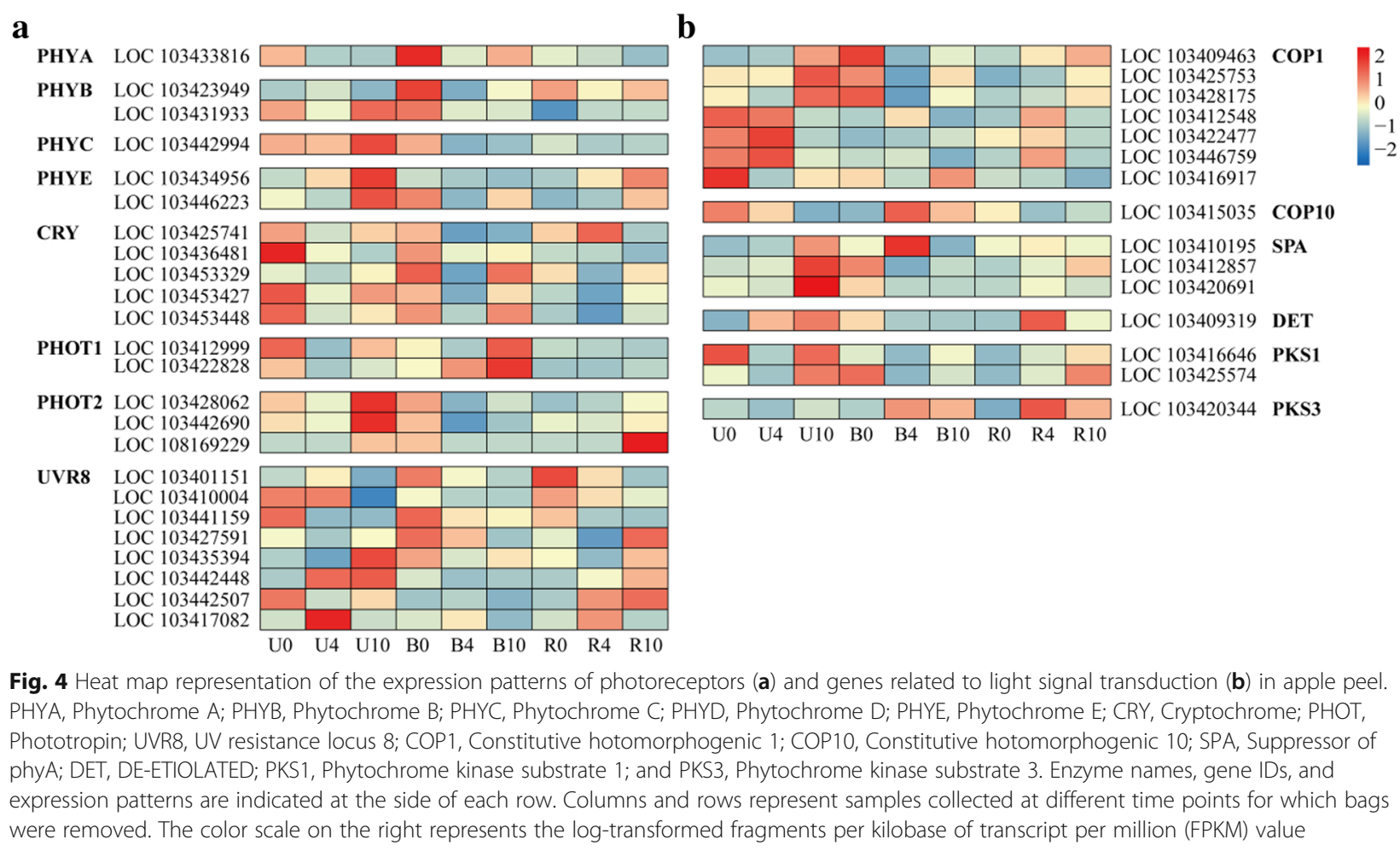

the 27 samples (Fig. 7b). Therefore, genes linked to this module may play crucial roles in anthocyanin accumulation in apple peel after bag removal, and their collective repression would likely lead to loss of color in bagged fruits. In this module ('darkolivegreen 4'), we found 22 structural genes and one MYB1 involved in anthocyanin biosynthesis and transportation. Cytoscape representation of these 23 genes with a WGCNA edge weight $\geq 0.80$ showed that they were highly connected; 21 of the 23 genes had 20 or more edges, and only two (DFR, LOC103416223; F3'H, LOC103418200) had a low number of edges (15 and 8, respectively; Fig. 7c).

Expression of genes involved in anthocyanin biosynthesis In total, we identified 48 transcripts of genes potentially participating in various steps of anthocyanin metabolic pathways (Fig. 8). In the earlier stages of the anthocyanin biosynthesis pathway, transcriptional levels of the structural genes PAL, 4CL, CHS, CHI, F3H, and F3'H were significantly upregulated in the $\mathrm{R}$ group. Expression of the two PALs was low in the $\mathrm{U}$ and $\mathrm{B}$ groups, while levels gradually increased in the $\mathrm{R}$ group. Three $4 C L$ genes exhibited highest levels in R10, and the other two peaked in R4. Transcriptional levels of three CHSs peaked in R10, and two CHIs increased gradually and peaked in R4, while LOC103454421 peaked in R10. Two F3Hs and two F3'Hs peaked in R10, while transcriptional levels of another two F3'Hs were highest in R4.
The later stages of the anthocyanin biosynthesis pathway mainly involved DFR, ANS, and UFGT (Fig. 8). Among DFRs, LOC103416223 and LOC103450464 were highly expressed and peaked in R10, while the highest expression of LOC103418902 occurred in R4. ANSs, UFGTs, and five MYB1s displayed similar expression patterns; they were highly expressed in the $\mathrm{R}$ group and levels peaked in R10. The other two MYB1s and seven bHLHs reached their highest expression levels in R4. Expression of two bHLHs peaked in R0. Two WD40s displayed high expression levels in U4, but with low expression in the $\mathrm{R}$ group. These data indicate most of the genes involved in anthocyanin biosynthesis play positive roles in the red pigmentation phenotype of 'Granny Smith' after bag removal.

\section{Function of the MdMYB1 promoter in transgenic tobacco and apple}

To test the function of the MdMYB1 promoter, three promoter fragments (2026 bp, complete sequence; 1869 $\mathrm{bp}$, lacking the -2026 to $-1870 \mathrm{bp}$ region; and $1927 \mathrm{bp}$, lacking the -1062 to $-964 \mathrm{bp}$ region) were transcriptionally fused to the promoterless reporter gene of GUS and each construct was introduced into tobacco leaves and apple peel. Compared with the complete promoter (pMYB1), GUS activity with pMYB1-2026 (MdMYB1 promoter lacking the -2026 to $-1870 \mathrm{bp}$ region) was decreased by 44.9 and $62.6 \%$ in tobacco leaves and apple 


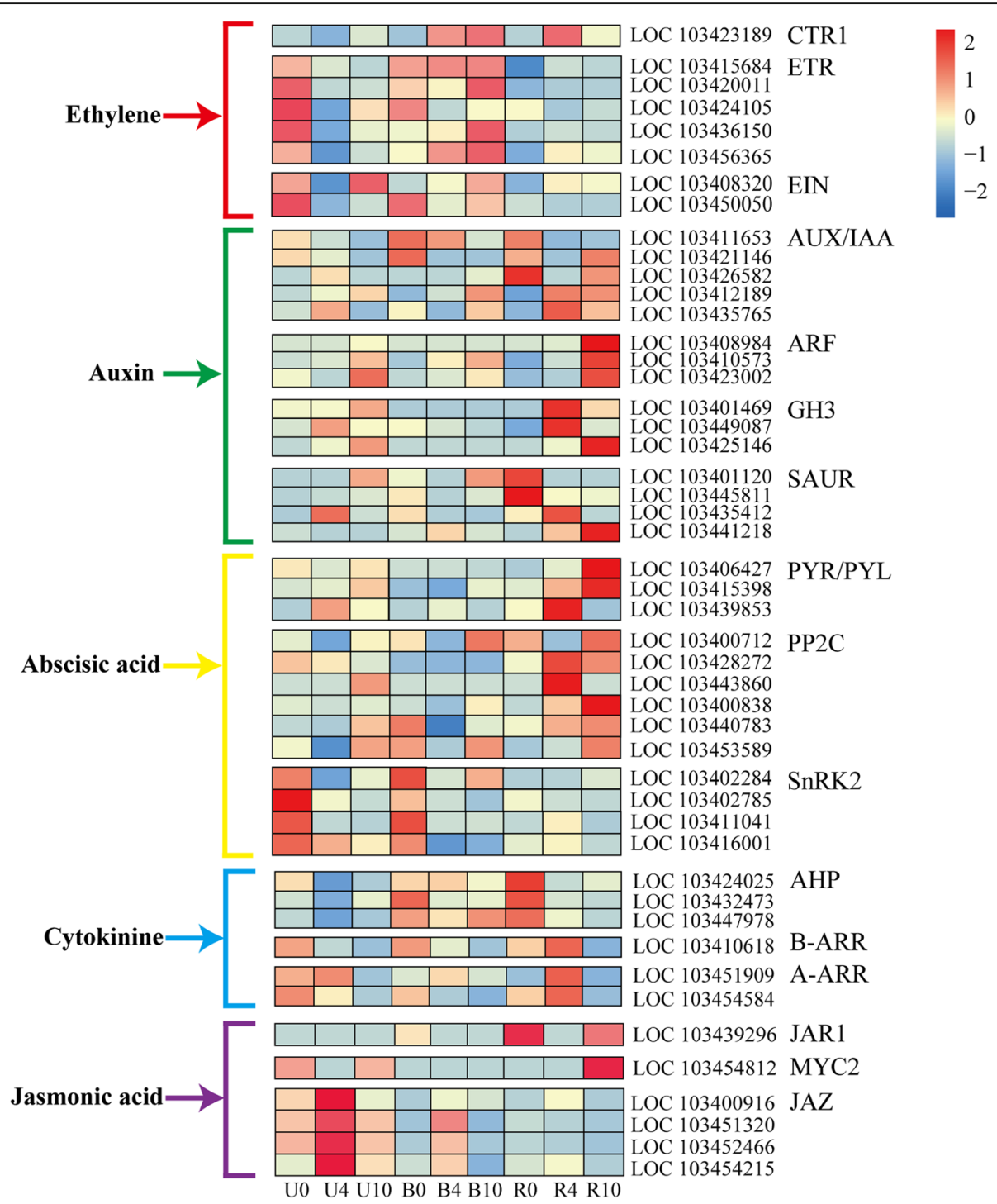

Fig. 5 DEGs mapped to plant hormone signal transduction pathways in the KEGG database. CTR1, serine/threonine-protein kinase CTR1; ETR, ethylene receptor; EIN, ethylene-insensitive protein; AUX, auxin influx carrier; IAA, auxin-responsive protein IAA; ARF, auxin response factor; GH3, auxin responsive GH3 gene family; SAUR, small auxin up RNA; PYR/PYL, abscisic acid receptor PYR/PYL family; PP2C, protein phosphatase 2C; SnRK2, serine/threonine-protein kinase SRK2; AHP, histidine-containing phosphotransfer peotein; B-ARR, two-component response regulator ARR-B family; A-ARR, two-component response regulator ARR-A family; JAR1, jasmonic acid-amino synthetase; MYC2, transcription factor MYC2; and JAZ, jasmonate ZIM domain-containing protein. Columns and rows in the heat map represent samples collected at different time points for which bags were removed. The color scale on the right represents the log-transformed FPKM value

peel, respectively; with pMYB1-1062 (MdMYB1 promoter lacking the -1062 to -964 bp region), the decrease was $43.3 \%$ in tobacco leaves and $59.2 \%$ in apple peel (Fig. 9b and d). The highest levels of GUS activity in tobacco leaves and apple peel were obtained with the pCAMBIA1031 (empty plasmid) construct. Additionally, fruits infiltrated with pMYB1 displayed enhanced pigmentation and increased anthocyanin accumulation around the injection sites, while pMYB1-2026 and pMYB1-1062 inhibited both anthocyanin accumulation and apple coloration (Fig. 9c, e and f). The anthocyanin content in apple peel treated with pMYB1-2026 and pMYB1-1062 was 14.7 and $12.4 \%$, respectively, relative to pMYB1 (100\%; Fig. 9f). These results indicate that pMYB1-2026 and pMYB1-1062 are essential for the increased accumulation of anthocyanins in apple peel after bag removal.

\section{Quantitative real-time PCR (qRT-PCR) validation of DEGs}

To validate the RNA-seq transcriptome results, 15 DEGs were selected for measurement of transcript levels by qRT-PCR. These genes included CHI, PAL, F3H, CHS, 


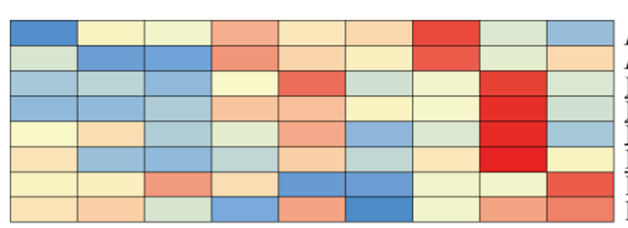

LOC 103432912 LOC 103453882

LOC 103426423

OC 103426425

LOC 1034449311

LOC 103449311

LOC 103451457

SOD

\begin{tabular}{l|l} 
LOC 103412103 & CAT
\end{tabular}

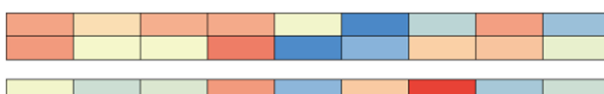

LOC 103429547

LOC 103415599

LOC 103415948

LOC 103418662

LOC 103434109

LOC 103451277

LOC 103408778

LOC 103410608

LOC 103412502

LOC 103413735

LOC 103423382

LOC 103429715

LOC 103432891

\begin{tabular}{l} 
LOC 103433738 \\
\hline LOC 103437839
\end{tabular}

POD

LOC 103447832

LOC 103448533

LOC 103406430

LOC 103440557

LOC 103442129

LOC 103442282

LOC 103442282

LOC

LOC 103444775

LOC 103447309

LOC 103454745

LOC 103414848

LOC 103404791

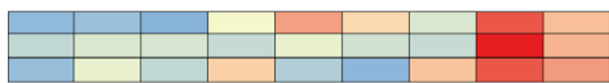

LOC 103410608

LOC 103413735

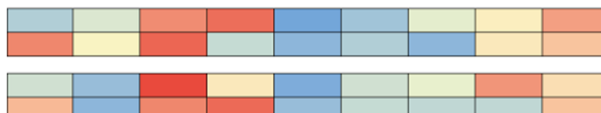

LOC 103443500

LOC 103456002

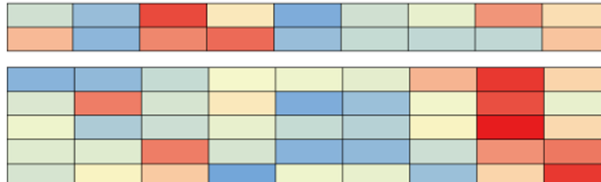

LOC 103411764

LOC 103404333

| MDHAR

LOC 103412502

LOC 103429715

LOC 103454744

LOC 103442282

GPX

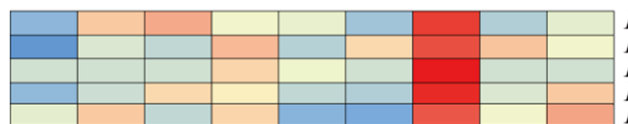

LOC 103452956

APX

(OC 103452956

LOC 103454097

LCC 103405151

LOC 103407647

LOC 103409159

LOC 103409925

LOC 103414311

LOC 103415530

LOC 103427104

LOC 103432552

LOC 103433528

LOC 103403298

LOC 103417250

LOC 103423404

LOC 103424010

GST

LOC 103426052

LOC 103435649

LOC 103436762

LOC 103443164

LOC 103445446

LOC 103451886

LOC 103452346

LOC 103452974

LOC 103405148

LOC 103432314

LOC 103432551

LOC 103427433

LOC 103443165

LOC 103449256

$\begin{array}{lllllllll}\mathrm{U} 0 & \mathrm{U} 4 & \mathrm{U} 10 & \mathrm{~B} 0 & \mathrm{~B} 4 & \mathrm{~B} 10 & \mathrm{R} 0 & \mathrm{R} 4 & \mathrm{R} 10\end{array}$

Fig. 6 (See legend on next page.) 
(See figure on previous page.)

Fig. 6 Heat map representation of the expression of genes related to antioxidant systems in apple peel. SOD, superoxide dismutase; CAT, catalase; POX, peroxidase; APX, ascorbate peroxidase; GR, glutathione reductase; MDHAR, monodehydroascorbate reductase; GPX, glutathione peroxidase; GST, glutathione S-transferase. Italicized, underlined, and bold text indicates DEGs exhibiting highest expression in R0, R4, and R10, respectively. Columns and rows in the heat map represent samples collected at different time points for which bags were removed. The color scale on the right represents the log-transformed FPKM value

$D F R$, UFGT, and ANS involved in anthocyanin biosynthesis; $M Y B 1$ that regulates anthocyanin biosynthesis; PKS1, CRY, COP1, and PHYA that are associated with photoreception and light signal transduction; $A R F$ that is involved in the auxin transduction pathway; and $S O D$ and GST antioxidant enzymes. The exact fold change of DEGs at several data points varied between RNA-seq and qRT-PCR methods (Fig. 10a). However, the overall expression patterns were strongly consistent (Pearson correlation coefficient $R^{2}=0.8188$; Fig. 10b), confirming the reliability of the RNA-seq results.

\section{Discussion}

Light-induced anthocyanin accumulation is mediated by signal transduction pathways in 'Granny Smith'

Light plays vital roles in regulating anthocyanin biosynthesis, especially in fruit peel $[8,37]$. In the current study, we observed red pigmentation in the green-skinned 'Granny Smith'

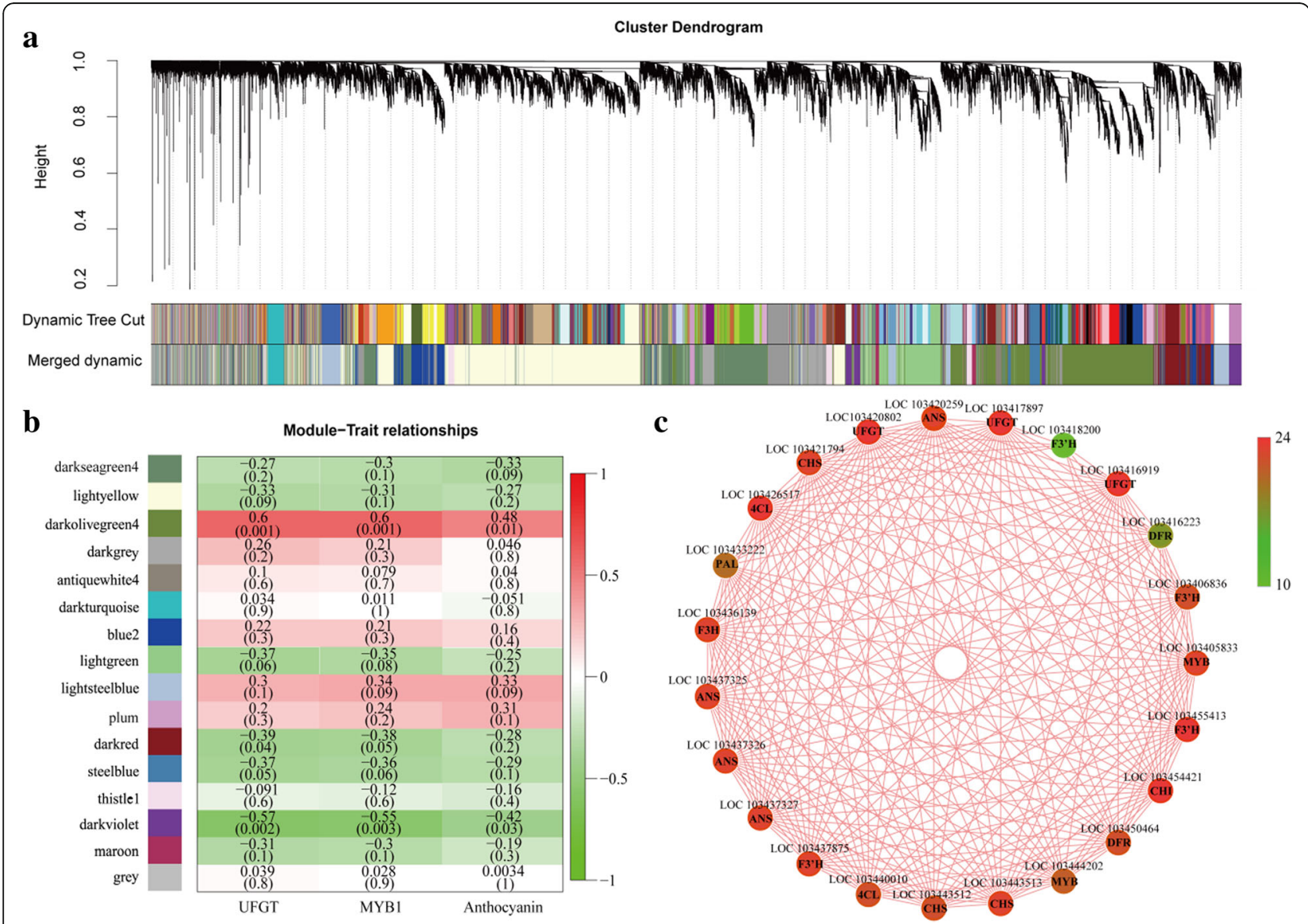

Fig. 7 Weighted gene co-expression network analysis (WGCNA) of DEGs identified in apple peel after bag removal. a Hierarchical cluster tree showing 16 modules of co-expressed genes. Each of the 18,152 DEGs is represented by a leaf in the tree, and each of the modules by a major tree branch. The lower panel shows modules in designated colors. b Module-trait correlations and corresponding $p$-values (in parentheses). The left panel shows the 16 modules and the number of member genes. The color scale on the right shows module-trait correlations from -1 (green) to +1 (red). The left panel labeled 'UFGT' represents expression changes of MdUFGT, which encodes the enzyme that catalyzes the last step in anthocyanin biosynthesis, as a trait. The middle panel labeled 'MYB1' represents expression changes of MdMYB1, the key transcriptional factor activating anthocyanin biosynthesis, as a trait. The right panel labeled 'Anthocyanin' represents anthocyanin biosynthesis as a trait. c Cytoscape representation of co-expressed genes with edge weights $\geq 0.80$ in the 'darkolivegreen 4 ' module. The edge number of genes ranges from 10 to 24 (color-coded using the scale on the right, from green to red). Member gene IDs and common names are given 


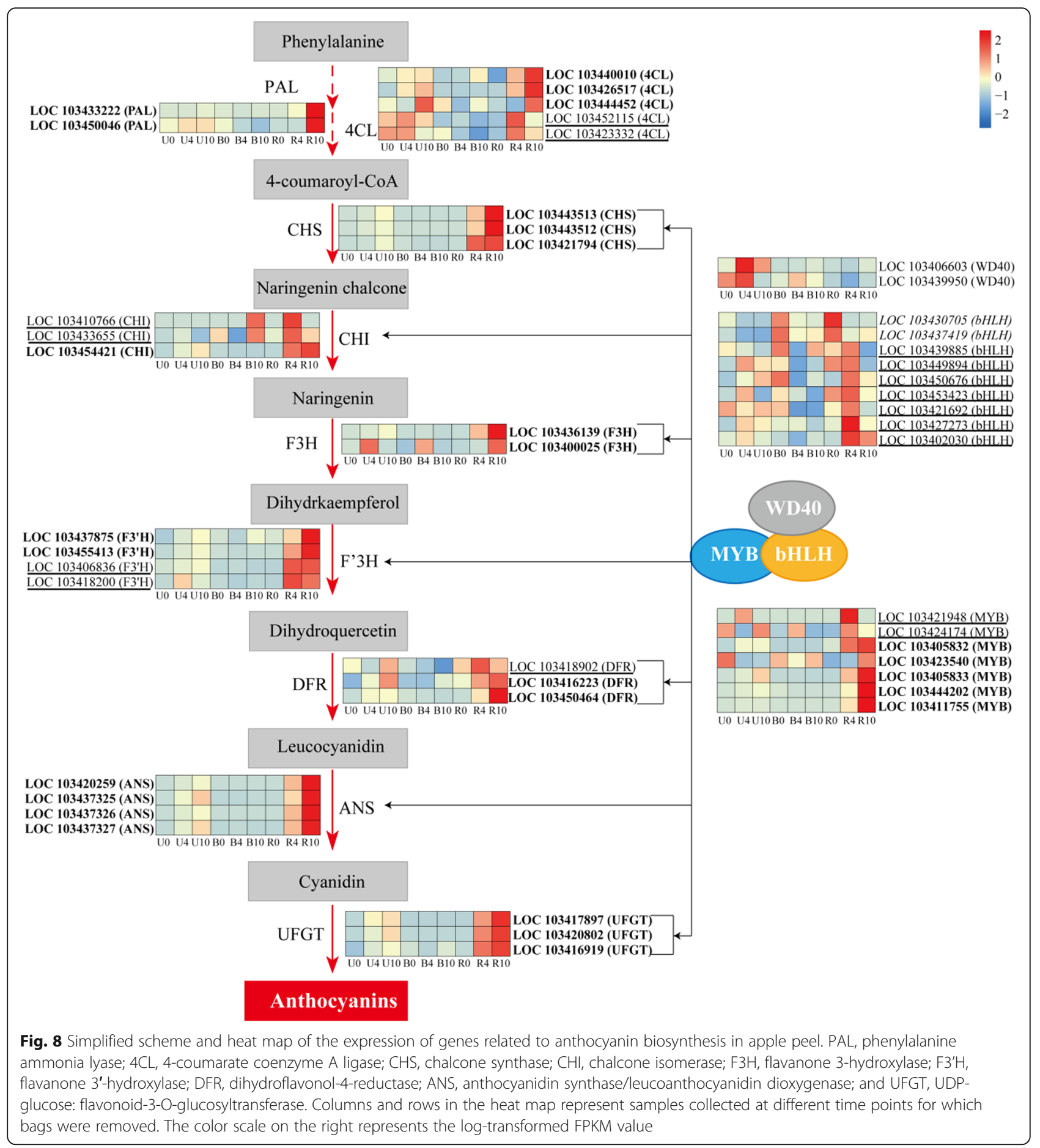

apple cultivar within a short time after bagging treatment, and anthocyanins were rapidly synthesized following bag removal (Fig. 1b). By contrast, natural and shaded conditions did not confer red pigmentation in 'Granny Smith' (Fig. 1a). This result indicates that bagging-induced coloration of non-red apples is mainly mediated by light, similar to observations in previous studies on lychee (Litchi chinensis) [15] and pear (Pyrus pyrifolia) [38]. Moreover, KEGG analysis showed that the genes involved in flavonoid biosynthesis were upregulated in the bag-removed group throughout the entire time course, further indicating the major effect of light on apple color.

Plants utilize multiple sensory photoreceptors to coordinate their responses to ambient light [39]. When dark-grown apple fruits were re-exposed to sunlight, we found two PHYEs, one PHOT2, and four UVB8s that 


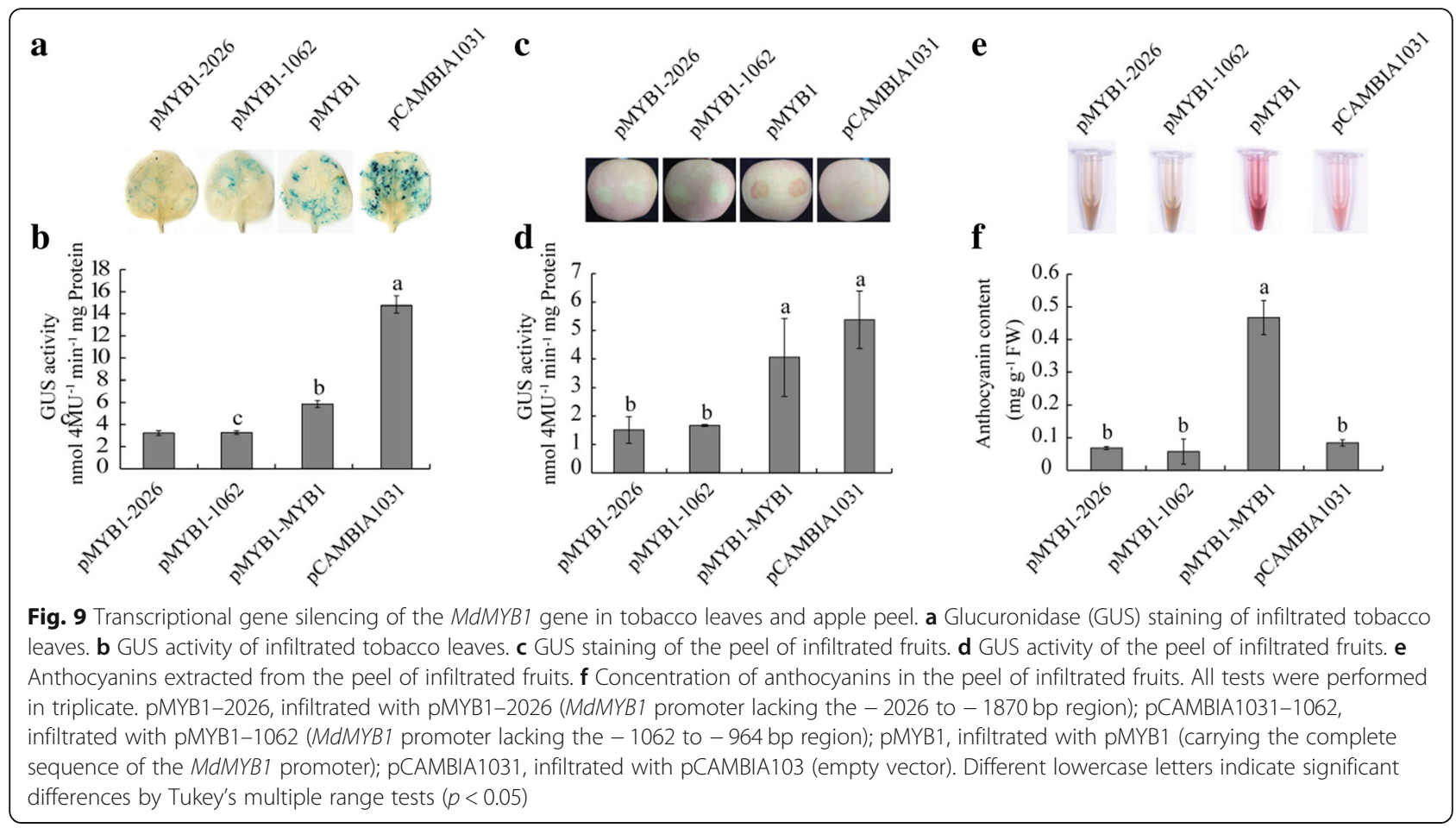

were markedly upregulated in apple peel, with levels peaking in the bag-removed group at 10 DABR (R10; Fig. 4a). These gene expression patterns are consistent with the trend of anthocyanin accumulation we observed in apple peel (Fig. 1b). We suspect that PHYEs, PHOT2, and $U V B 8$ s participate in the regulation of anthocyanin biosynthesis in the peel of 'Granny Smith' apples. Among these photoreceptors, UVR8s may be the most important photoreceptor in the pericarp of lychee [15].

COP1s are required for the ubiquitination and degradation of the MdMYB1 protein in the dark [17], which is a positive regulator for fruit coloration [8]. In the present study, we found that expression of COP1s was generally low in the bag-removed group, although six COP1s were upregulated in this group; this result reflect the differential expression of COP1 in response to light in 'Granny Smith' apples. In addition, upregulation of one DET, two PKS1s, and one PKS3 in the bag-removed group (Fig. 4b) reflects their possible involvement in the regulation of anthocyanin accumulation in 'Granny Smith' apple fruits after bag removal. These results indicate that the bag-removed fruits perceive and transduce light signals to regulate anthocyanin accumulation in 'Granny Smith' after bag removal.

\section{Plant hormones are involved in bagging-induced apple coloration}

The plant hormone signaling pathways can be affected by light $[16,38]$. ETR is a receptor of the plant hormone ethylene. Here we found that expression of ETRs was lower in apple peels of the bag-removed group compared with unbagged and bagged groups (Fig. 5). Our result is consistent with ethylene suppression of anthocyanin accumulation via binding to ETRs in Arabidopsis [40] and tobacco (Nicotiana tabacum) [41]. CTR and EIN function in a wide range of ethylene responses in plants $[42,43]$. In the present study, increased CTR expression and decreased EIN expression were found in the bag-removed group (Fig. 5). These distinct expression patterns indicate that ethylene signal has different functions in the regulation of anthocyanin biosynthesis in apple peel through various transduction pathways.

Endogenous application of auxins can retard anthocyanin accumulation in grape (Vitis vinifera) [44]. However, we observed significant upregulation of genes encoding auxin-responsive elements, such as AUX/IAAs, ARFs, GH3s and SAURs in the bag-removed group (Fig. 5), which implies that auxins in vivo play positive roles in bagging-induced anthocyanin biosynthesis in apple peel. In addition, abscisic acid treatment can increase anthocyanin accumulation in cherry (Prunus avium L.) [45], grape (Vitis vinifera) [46], and strawberry (Fragaria ananassa Duch.) [47]. By contrast, abscisic acid also has a repressive effect on anthocyanin biosynthesis in Arabidopsis [48]. In the present work, we observed increased transcriptional levels of several PYRs/PYLs and PP2Cs, and low expression of $S n R K 2 s$ in the bag-removed group (Fig. 5). This result indicates that abscisic acid is involved in bagging-induced anthocyanin biosynthesis via altering expression patterns of particular genes. 


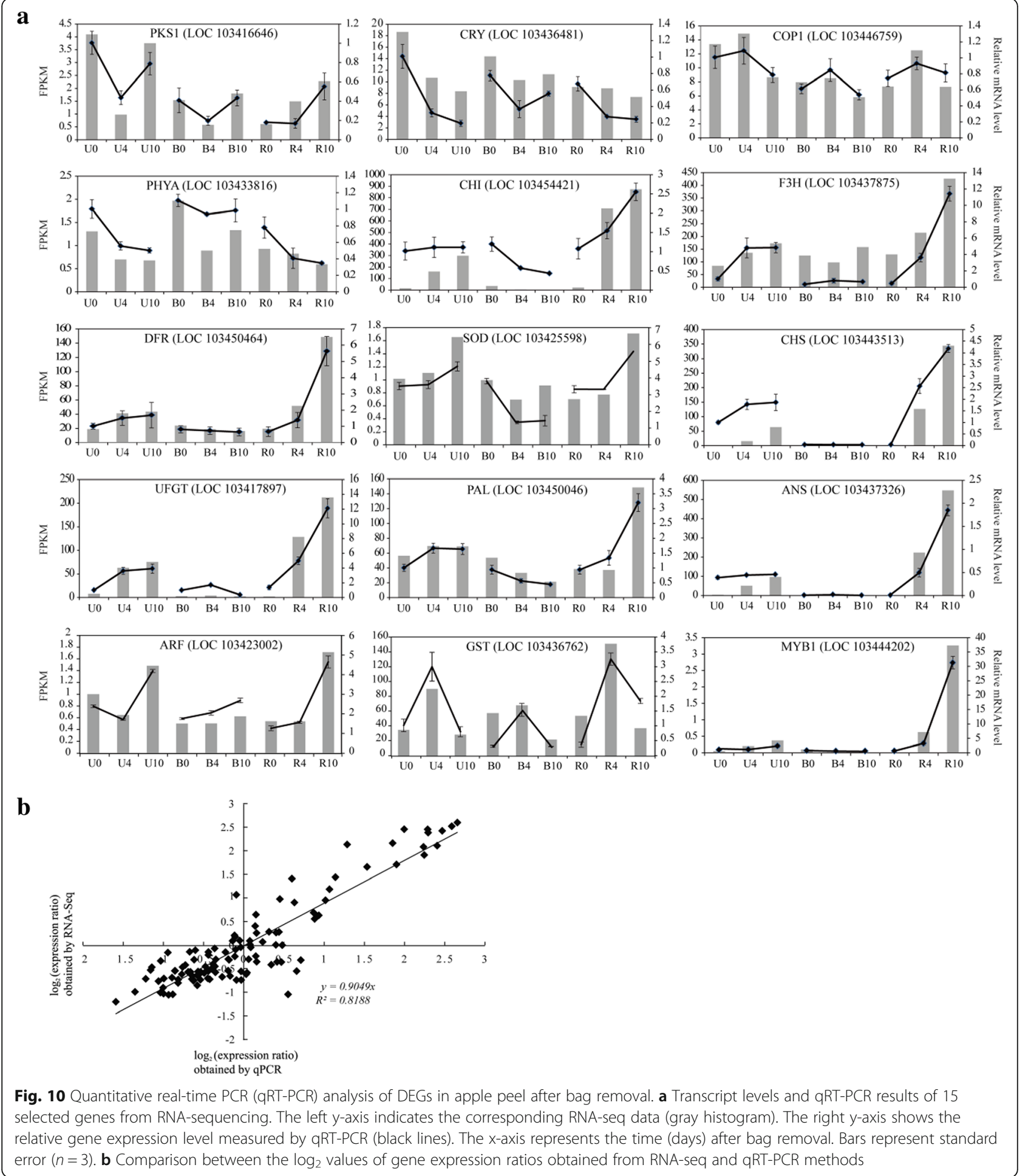

Cytokinins are positive regulators of the anthocyanin biosynthesis pathway and they function by the modulation of PAL, CHI, CHS, and DFR expression in Arabidopsis [49]. Cytokinin signaling is mediated by AHPs, $B$-ARRs, and A-ARRs in Arabidopsis [50]. Herein, we found that expression of most AHPs was decreased in the bag-removed group, in contrast to the upregulation of $B-A R R$ and two A-ARRs in this group (Fig. 5). This result allows us to postulate that cytokinins are part of a complicated system regulating anthocyanin accumulation in apple peel. Furthermore, JAZ proteins directly interact with $b H L H s$ and MYBs in the MBW complex 
[51]. However, we found four JAZs that were downregulated in the bag-removed group (Fig. 5), indicating an increase in JAZ protein does not affect the expression or function of essential bagging-inducible anthocyanin-related MYBs in apple peel [38]. Taken together, our results indicate that plant hormones play crucial roles in the regulation of fruit coloration in 'Granny Smith' after bag removal. In plants, light-dependent regulation of anthocyanin formation can be regulated via the interactions among different plant hormones [25]. Exactly how these hormones are involved in fine-tuning the regulation of light-induced anthocyanin biosynthesis and the interactions among them remain to be elucidated.

\section{ROS scavenging enzymes in apple peel are altered after bag removal}

Production of ROS in plants causes oxidative stress [52], and various photoprotective mechanisms in red and non-red apple peel operate under high light conditions [33]. In this study, differences in the expression of antioxidant enzyme genes were evident across the three treatment groups, reflecting the dynamic responses of apple peel to sunlight exposure.

SOD provides the first line of defense against the toxic effects of elevated ROS levels [32]. Here we found, that $S O D s$ were upregulated in the bag-removed group (Fig. 6), indicating their involvement in combating oxidative stress in apple peel in response to sunlight exposure. Although transcription of $C A T s$ was upregulated in the bag-removed group, one $C A T$ gene was highly expressed in the bagged group (Fig. 6). We suspect that CAT isozymes may be regulated temporally and spatially, and may respond differentially to light in apple [53, 54]. Moreover, expression of APXs and PODs was increased when dark-grown apples were exposed to light (Fig. 6). APX may increase POD activity, thereby enhancing the ROS scavenging system and improving the oxidative stress tolerance [55].

Interestingly, expression of one $G R$ and one MDHAR was higher in the unbagged group at 10 DABR (U10) and bagged group at 0 DABR (B0), compared with the bag-removed group, in response to long-term sunlight exposure and long-term darkness, respectively (Fig. 6). Thus, these proteins may play crucial roles in plant responses to various stresses [56, 57]. Overexpression of GPX enhances plant tolerance to various abiotic stresses $[58,59]$. In the present study, significantly increased expression of GPXs was observed in the bag-removed group (Fig. 6), indicating their possible involvement in responding to the transition from long-term darkness to light exposure in apple. Additionally, expression levels of GSTs were increased in the bag-removed group than the other two groups (Fig. 6), which is consistent with the previous finding of GST family members mediating anthocyanin transport in 'Red Bartlett' pears (Pyrus communis L.) [60]. Thus, when dark-grown apple fruits were re-exposed to sunlight, transcriptional levels of some antioxidant enzyme genes increased (Fig. 6), with their levels being correlated with anthocyanin accumulation in fruit peel (Fig. 1b). We speculate that the higher expression of antioxidant enzyme genes is involved in anthocyanin accumulation in 'Granny Smith' after bag removal. However, the mechanisms of the enzymatic antioxidant systems responsible for anthocyanin biosynthesis in apple peel following bagging treatment require further analysis.

\section{A WGCNA module is strongly associated with anthocyanins, and its implications}

The transcriptomes of unbagged (green peel), bagged (white peel), and bag-removed (red peel) groups were characterized to identify DEGs, and thereby gain insight into the molecular mechanisms underpinning the regulation of anthocyanin biosynthesis and accumulation in apple peel. The identified DEGs likely represent the genes most closely associated with anthocyanins because they not only include DEGs among the treatment groups at three developmental stages, but also cover DEGs between two adjacent stages within each treatment group. The results obtained thus account for color progression (in bag-removed group) that may not be captured by direct comparison of unbagged, bagged, and bag-removed groups. The WGCNA network of DEGs revealed the 'darkolivegreen 4' module containing 23 genes highly correlated with anthocyanin content (Fig. 7); thus upregulation of genes in this module may result in red pigmentation in apple fruits after bag removal. Examining these 23 genes in the three treatment groups demonstrated that their expression was significantly correlated with anthocyanin content in apple peel following bag removal (Figs. 8 and 1b); this indicates these genes may play critical roles in red pigmentation in the 'Granny Smith' cultivar after bagging treatment.

Furthermore, we identified other structural and transcription factor-encoding genes in apple peel. Notably, structural genes generally exhibited a similar expression pattern to those of MYBs in apple fruits after bag removal (Fig. 8). Our result further indicats that $M d M Y B$ plays an positive role in anthocyanin accumulation in apple peel following bag removal $[34,36]$. The -2026 to $-1870 \mathrm{bp}$ and -1062 to $-964 \mathrm{bp}$ regions of the MdMYB1 promoter comprise MYB-binding sites and light-responsive elements. Hypomethylation of these two regions is associated with pigmentation in 'Granny Smith' apples after bag removal [11]. Here deletion of these two regions of the $M d M Y B 1$ promoter induced a significant decrease in GUS activity and anthocyanin accumulation (Fig. 9); thus, these regions are essential for 
MdMYB1-mediated regulation of anthocyanin accumulation and pigmentation in the 'Granny Smith' cultivar. Since many genes in the WGCNA module were not characterized, this study not only provides new insight into the anthocyanin biosynthesis pathway, but also presents a list of candidate genes for more dedicated functional studies in the future.

\section{Conclusions}

In this study, the green skinned 'Granny Smith' apple cultivar underwent red pigmentation after bagging treatment, while unbagged and bagged fruits did not change color. Following bag removal, expression of $P H Y E$, PHOT2, UVB8, DET, PKS1, PKS3, and COP1 involved in light signal perception and transduction was tightly correlated with anthocyanin biosynthesis in apple peel. Additionally, hormone signaling-related genes might be involved in bag-induced red pigmentation through differences in expression patterns. Furthermore, most antioxidant genes including SOD, CAT, POD, APX, GPX, and GST were upregulated. The red pigmentation of 'Granny Smith' apples is likely a consequence of the collective upregulation of 23 key genes that form the 'darkolivegreen 4' module in the WGCNA network. In particular, $M d M Y B 1$ is one of the genes most upregulated in 'Granny Smith' after bag removal, and the 2026 to $-1870 \mathrm{bp}$ and -1062 to -964 bp regions of this promoter element are essential for MdMYB1-mediated regulation of anthocyanin accumulation and red pigmentation in the 'Granny Smith' apple cultivar. The transcriptome data and identified DEGs provide valuable information and candidate genes for investigating the mechanisms regulating the coloration of 'Granny Smith' and other non-red apple cultivars.

\section{Methods}

\section{Plant materials and experimental treatments}

This work investigated the mechanisms underpinning red pigmentation in 'Granny Smith' after bag removal, based on transcriptome sequencing. 'Granny Smith' cultivar fruits were collected from the Baishui Apple Experimental Station of Northwest A\&F University in Yangling, Shaanxi Province, China $\left(35^{\circ} 21^{\prime} \mathrm{N}, 109^{\circ} 55^{\prime} \mathrm{E}\right.$, elevation $=850 \mathrm{~m}$ ). Trees were 6 years old, grafted on the M26 rootstock (Malus domestica), and planted at a density of $4 \times 2 \mathrm{~m}$. Bagging was applied as described previously [11]. Briefly, all fruits were wrapped in a paper bag (Hongtai, Shanxi, China) at 40 DAFB, and bags were removed at 160 DAFB (bag-removed group). Fruits without bagging treatment (unbagged group) and dark-grown apple fruits (bagged group) were used for comparison. Fruit samples were randomly taken at $0,2,4,6,8$, and 10 days after bag removal (DABR). Each point-in-time sample consisted of 12 fruits, and three biological replicates were harvested, with at least four fruits collected from two trees per replicate. Fruit peel was collected with a peeler, immediately frozen in liquid nitrogen and stored at $-80^{\circ} \mathrm{C}$. Peel samples from 0,4 , and 10 DABR were used for transcriptome sequencing and gene expression analysis.

For deletion analysis of the MdMYB1 promoter, 'Pink lady' apple fruits were bagged at $40 \mathrm{DAFB}$, and bagged fruits were collected at 160 DAFB. Tobacco (Nicotiana benthamiana) plants were grown in vitro at $25^{\circ} \mathrm{C}$ on Murashige-Skoog medium [61].

\section{Anthocyanin and chlorophyll measurement}

Measurement of anthocyanin content was performed as described previously [35]. Apple peel $(0.5 \mathrm{~g})$ was finely ground in $5 \mathrm{~mL} \mathrm{HCl} /$ methanol $(1 / 99, \mathrm{v} / \mathrm{v})$, and then centrifuged at $13,000 \times g$ for $10 \mathrm{~min}$. A high-performance liquid chromatography instrument with a photodiode array detector (Waters, Milford, USA) was used for analysis. Separation of anthocyanins was accomplished on a C18 column $(5 \mu \mathrm{m}$ internal diameter, $250 \times 4.6 \mathrm{~mm}$; Waters). Cyanidin 3-galactoside served as a standard (Sigma-Aldrich, St. Louis, USA). Chlorophyll was extracted using a UV/visible spectrophotometer (UV-2550, Shimadzu Corp., Kyoto, Japan) as described previously [62]. Three independent biological replications were performed for each experiment.

\section{RNA extraction, library preparation, and RNA-seq}

Total RNA was extracted from fruit peel using the TRIzol RNA plant plus reagent (Tiangen, Beijing, China) following the manufacturer's instructions. RNA quality was assessed on an Agilent 2100 Bioanalyzer (Agilent Technologies, Palo Alto, USA). Samples with an RNA integrity number score $>7.5$ were selected for deep sequencing. Next, mRNAs were enriched and cleaved into small pieces, which served as templates for cDNA synthesis. Purification of the cDNA fragments and PCR amplification were performed as described previously [35]. In total, nine sets of raw reads were obtained, corresponding to the three treatment groups at $0(\mathrm{U} 0 / \mathrm{B} 0 / \mathrm{R} 0), 4(\mathrm{U} 4 / \mathrm{B} 4 / \mathrm{R} 4)$, and 10 (U10/B10/R10) DABR. To verify the reproducibility of the sequencing data, we calculated Pearson's correlation coefficients for three biological replicates at each developmental stage using the $\log _{10}$ fragments per kilobase of transcript per million mapped reads (FPKM) method [63].

\section{RNA-seq data processing and mapping of reads to the apple genome}

Raw read processing of transcript datasets was carried out by Genedenovo Biotechnology Co., Ltd. (Guangzhou, China). Raw reads were further filtered according to the method of Ma et al. [35]. All clean reads were mapped to the apple $(M$. domestica) genome sequence [64] using 
SOA-Paligner/soap2 [65]. Clean reads were then aligned with the reference genome and transcripts were reconstructed using Cufflinks [66].

\section{Identification of DEGs}

All clean reads were aligned to genes using TopHat2 [67] and then normalized into FPKM reads [63]. DEGs among different treatment groups at each of the three developmental stages were identified, along with DEGs between adjacent stages within each group. The original $p$-values were adjusted using the Benjamini and Hochberg approach [68] to minimize the false discovery rate (FDR). DEGs with $\mid$ fold change $\mid \geq 2$ and FDR $<0.05$ were considered significant.

\section{Functional annotation}

For Gene Ontology (GO) term enrichment analysis, all DEGs were mapped to GO terms in the GO database (http://www.geneontology.org/) [69]. KEGG pathway enrichment analysis was performed using the KEGG database (http://www.genome.jp/kegg/) [70]. Significant GO/ KEGG enrichment compared with the genomic background was determined using hypergeometric tests. Calculated $p$-values were subjected to FDR correction, and FDR $\leq 0.05$ was applied as the threshold.

\section{Construction of co-expression networks}

Co-expression network analysis was performed using an $\mathrm{R}$ package for WGCNA [71]. WGCNA network construction and module detection was conducted using an unsigned type of topological overlap matrix, a power $\beta$ of 10 , a minimal module size of 50 , and a branch merge cut height of 0.7 . The module eigengene (the first principal component of a given module) value was calculated and used for evaluation of the association between modules and anthocyanin content in the 27 samples. The most significant module ('darkolivegreen 4') based on 23 genes with a WGCNA edge weight $>0.80$ was represented using Cytoscape 3.3.0 [72]. Expression data were clustered using Cluster 3.0 [18] and displayed using Java Treeview [73].

\section{Vector construction and transformation in tobacco and apple} To analyze the function of the $M d M Y B 1$ promoter, a $2026 \mathrm{bp}$ promoter fragment (the complete sequence), a 1869 bp fragment (MdMYB1 promoter lacking the -2026 to $-1870 \mathrm{bp}$ region), and a $1927 \mathrm{bp}$ fragment (MdMYB1 promoter lacking the -1062 to $-964 \mathrm{bp}$ region) were cloned from genomic DNA extracted as described previously [11]. Overlap extension PCR was used according to Wurch et al. [74]. Specific PCR primer information is listed in Additional file 2: Tables S4 and S5. After digestion with restriction enzymes $\mathrm{XbaI}$ and $\mathrm{NcoI}$, the three promoter fragments were subcloned into the binary plasmid pCAMBIA1031 to replace the CaMV35S promoter, resulting in a series of pMYB1-del promoter::glucuronidase (GUS) fusion expression vectors, namely pMYB1-2026 (MdMYB1 promoter lacking the -2026 to - 1870 bp region), pMYB1-1062 (MdMYB1 promoter lacking the -1062 to $-964 \mathrm{bp}$ region), and pMYB1 (complete sequence of the MdMYB1 promoter). The empty pCAMBIA1031 plasmid served as a control.

Inoculation of tobacco leaves and apple peel with Agrobacterium tumefaciens strain EHA105 cells was conducted as previously described [17, 75]. Four expression vectors, pCAMBIA1031, pMYB1-2026, pMYB1-1062, and pMYB1, were injected into tobacco leaves and bagged apple fruit peel using a needleless syringe. Injected samples were incubated overnight in the dark at room temperature, then exposed to white light $\left(540 \mu \mathrm{mol} \cdot \mathrm{m}^{-2} \cdot \mathrm{s}^{-1}\right)$ with a $16 \mathrm{~h}$ photoperiod at $25^{\circ} \mathrm{C}$ in a growth chamber. After 2 weeks, the anthocyanin content in injected apples was determined using a UV-2550 $\mathrm{UV} /$ visible spectrophotometer as previously described [76].

\section{Histochemical and fluorimetric GUS activity analysis}

For histochemical staining of GUS, excised tobacco leaf and apple peel discs were immediately treated with $1 \mathrm{mM}$ 5-bromo-4-chloro-3-indolyl-b-D-glucuronide in $100 \mathrm{mM}$ phosphate buffer $\mathrm{pH} 7.0,10 \mathrm{mM}$ EDTA, $0.5 \mathrm{mM}$ potassium ferrocyanide, and $0.1 \%$ Triton $\mathrm{X}-100$ [77] at $37^{\circ} \mathrm{C}$ for $24 \mathrm{~h}$. Stained samples were bleached with $70 \%(\mathrm{v} / \mathrm{v})$ ethanol, and GUS activity was determined by measuring the fluorescence of 4-methylumbelliferone produced by GUS cleavage of 4-methylumbelliferyl- $\beta$-D-glucuronide (Sigma) as described previously [78]. The protein concentration in the supernatant was determined using the Bradford procedure with bovine serum albumin (Sigma) as a standard. Three independent biological replications were performed for each experiment.

\section{qRT-PCR analysis}

qRT-PCR analysis was performed according to Ma et al. [35]. Actin (GenBank: GQ339778.1) was used as a reference gene. Specific primers were designed using Primer 5 (Additional file 2: Table S6). Data were analyzed using the $2^{-\Delta \Delta C T}$ method [79].

\section{Statistical analysis}

All data were subjected to a one-way analysis of variance. Significant differences between group means were assessed by Tukey's post hoc tests $(p<0.05)$ using SPSS 16.0 Statistics (SPSS Inc., Chicago, IL, USA).

\section{Additional files}

Additional file 1: Table S1. Statistics related to the quality and output of RNA-seq libraries. Table S2. Throughput and quality of RNA-seq of differentially expressed genes libraries from "Granny Smith" apple peels. 
Table S3. Pearson's correlation coefficients of RNA-seq data for three biological replicates at each developmental stage. (XLSX $19 \mathrm{~kb}$ )

Additional file 2: Table S4. DNA sequences of oligonucleotide primers used in the MdMYB1 promoter sequence analysis. Table S5. DNA sequences of oligonucleotide primers used in the overlap extension PCR. Table S6. Primer sequences used in the quantitative real-time PCR. Figure S1. Clusters of differentially expressed transcripts with expression profile changes based on the number of genes and the significance of expression profiles. (DOCX $80 \mathrm{~kb}$ )

\section{Abbreviations}

4CL: 4-coumarate coenzyme A ligase; A-ARR: Two-component response regulator ARR-A family; AHP: Histidine-containing phosphotransfer protein; ANS: Anthocyanidin synthase/leucoanthocyanidin dioxygenase;

APX: Ascorbate peroxidase; ARF: Auxin response factor; AUX/IAA: Auxin influx carrier/auxin-responsive protein IAA; B: Bagged; B0/4/10: Bagged fruits at 0, 4, 10 DABR, respectively; B-ARR: Two-component response regulator ARR-B family; CAT: Catalase; CHI: Chalcone isomerase; CHS: Chalcone synthase; COP1: CONSTITUTIVE PHOTOMORPHOGENIC 1; CRY: Cryptochrome; DABR: Days after bag removal; DAFB: Days after full bloom;

DEG: Differentially expressed gene; DET: DE-ETIOLATED; DFR: Dihydroflavonol 4-reductase; F3H: Flavanone 3-hydroxylase; GH3: Auxin responsive GH3 gene family; GO: Gene Ontology; GPX: Glutathione peroxidase; GR: Glutathione reductase; GST: Glutathione-S-transferase; HY5: LONG HYPOCOTYL 5; JAR1: Jasmonic acid-amino synthetase; JAZ: Jasmonate ZIM domaincontaining protein; KEGG: Kyoto Encyclopedia of Genes and Genomes; MBW: MYB-bHLH-WD40/WDR; MDHAR: Monodehydroascorbate reductase; MYC2: Transcription factor MYC2; PAL: Phenylalanine ammonia lyase; PHOT: Phototropin; PHY: Phytochrome; PKS1: PHYTOCHROME KINASE SUBSTRATE 1; pMYB1: Complete sequence of the MdMYB1 promoter; pMYB1-1062: MdMYB1 promoter lacking the -1062 to -964 bp region; pMYB1-2026: MdMYB1 promoter lacking the -2026 to -1870 bp region; POD: Peroxidase; PP2C: Protein phosphatase 2C; PYR/PYL: Abscisic acid receptor; qRT-PCR: Quantitative real-time; R: Bag-removed; R0/4/10: Bagremoved fruits at 0, 4 and 10 DABR, respectively; ROS: Reactive oxygen species; SAUR: Small auxin up RNA; SnRK2: Serine/threonine protein kinase SRK2n; SOD: Superoxide dismutase; SPA: Suppressor of phyA; U: Unbagged; U0/4/10: Unbagged fruits at 0, 4, 10 DABR, respectively; UFGT: UDPglucose:flavonoid 3-O-glucosyltransferase; WGCNA: Weighted gene coexpression network analysis

\section{Acknowledgements}

The authors are grateful to Prof. Jidi Xu for critically reviewing the manuscript.

\section{Funding}

This study was funded by the National Key Research and Development Project (2016YFD0201131), the earmarked fund for the National Natural Science Foundation of China (31471845), and the Modern Agro-industry Technology Research System of China (CARS-27).

\section{Availability of data and materials}

The datasets generated and/or analyzed during the current study have been deposited in the NCBI Sequence Read Archive repository, with sample accession numbers SAMN07972647, SAMN07972648, SAMN07972649, SAMN07972650, SAMN07972651, SAMN07972652, SAMN07972653, SAMN07972654, SAMN07972655, SAMN07972656, SAMN07972657, SAMN07972658, SAMN07972659, SAMN07972660, SAMN07972661, SAMN07972662, SAMN07972663, SAMN07972664, SAMN07972665, SAMN07972666, SAMN07972667, SAMN07972668, SAMN07972669, SAMN07972670, SAMN07972671, SAMN07972672, and SAMN07972673 (https://www.ncbi.nlm.nih.gov/bioproject/416979). Any reasonable requests are available from the corresponding author.

\section{Authors' contributions}

$\mathrm{CM}$ and $\mathrm{ZZ}$ conceived the research; CM designed and performed the experiments, analyzed data and drafted the manuscript; BL and YY participated in coordination of the study; BC and YW analyzed histochemical and fluorimetric GUS activity; JY and LL performed identification of anthocyanin compounds; CM and ZZ wrote the paper. All authors read and approved the final version of the manuscript.

Ethics approval and consent to participate

Not applicable.

\section{Consent for publication}

Not applicable.

\section{Competing interests}

The authors declare that they have no competing interests.

\section{Publisher's Note}

Springer Nature remains neutral with regard to jurisdictional claims in published maps and institutional affiliations.

\section{Received: 17 December 2018 Accepted: 24 April 2019}

Published online: 09 May 2019

\section{References}

1. He J, Giusti MM. Anthocyanins: natural colorants with health-promoting properties. Annu Rev Food Sci T. 2010;1:163.

2. Vimolmangkang S, Zheng DM, Han YP, Khan MA, Soria-Guerra RE, Korban SS. Transcriptome analysis of the exocarp of apple fruit identifies lightinduced genes involved in red color pigmentation. Gene. 2014;534:78-87.

3. Van den Ende W, El-Esawe SK. Sucrose signaling pathways leading to fructan and anthocyanin accumulation: a dual function in abiotic and biotic stress responses? Environ Exp Bot. 2014;108:4-13.

4. Huang CH, Yu B, Teng YW, Su J, Shu Q, Cheng ZQ, Zeng LQ. Effects of fruit bagging on coloring and related physiology, and qualities of red Chinese sand pears during fruit maturation. Sci Hortic-Amsterdam. 2009;121:149-58.

5. Fischer TC, Gosch C, Pfeiffer J, Halbwirth H, Halle C, Stich K, Forkmann G. Flavonoid genes of pear (Pyrus communis). Trees-Struct Funct. 2007; 21:521-9.

6. Takos AM, Ubi BE, Robinson SP, Walker AR. Condensed tannin biosynthesis genes are regulated separately from other flavonoid biosynthesis genes in apple fruit skin. Plant Sci. 2006;170:487-99.

7. Honda C, Kotoda N, Wada M, Kondo S, Kobayashi S, Soejima J, Zhang Z, Tsuda T, Moriguchi T. Anthocyanin biosynthetic genes are coordinately expressed during red coloration in apple skin. Plant Physiol Bioch. 2002;40: 955-62.

8. Takos AM, Jaffé FW, Jacob SR, Bogs J, Robinson SP, Walker AR. Lightinduced expression of a MYB gene regulates anthocyanin biosynthesis in red apples. Plant Physiol. 2006;142:1216-32.

9. Lloyd A, Brockman A, Aguirre L, Campbell A, Bean A, Cantero A, Gonzalez A. Advances in the MYB-bHLH-WD repeat (MBW) pigment regulatory model: addition of a WRKY factor and co-option of an anthocyanin MYB for betalain regulation. Plant Cell Physiol. 2017:58:1431-41.

10. Xu Y, Feng S, Jiao Q, Liu C, Zhang W, Chen W, Chen X. Comparison of MdMYB1 sequences and expression of anthocyanin biosynthetic and regulatory genes between Malus domestica Borkh. cultivar 'Ralls' and its blushed sport. Euphytica. 2012;185:157-70.

11. Ma C, Jing C, Chang B, Yan J, Liang B, Liu L, Yang Y, Zhao Z. The effect of promoter methylation on MdMYB1 expression determines the level of anthocyanin accumulation in skins of two non-red apple cultivars. BMC Plant Bio. 2018;18:108.

12. Bai S, Tuan PA, Saito T, Honda C, Hatsuyama Y, Ito A, Moriguchi T Epigenetic regulation of MdMYB1 is associated with paper bagging-induced red pigmentation of apples. Planta. 2016;244:573-86.

13. Ban $Y$, Honda C, Hatsuyama Y, Igarashi M, Bessho H, Moriguchi T. Isolation and functional analysis of a MYB transcription factor gene that is a key regulator for the development of red coloration in apple skin. Plant Cell Physiol. 2007:48:958.

14. Davies C, Boss PK. Treatment of grape berries, a nonclimacteric fruit with a synthetic auxin, retards ripening and alters the expression of developmentally regulated genes. Plant Physiol. 1997;115:1155-61.

15. Zhang HN, Li WC, Wang HC, Shi SY, Shu B, Liu LQ, Wei YZ, Xie JH. Transcriptome profiling of light-regulated anthocyanin biosynthesis in the pericarp of Litch. Front Plant Sci. 2016;7:963.

16. Jaakola L. New insights into the regulation of anthocyanin biosynthesis in fruits. Trends Plant Sci. 2013;18:477-83. 
17. Li YY, Mao K, Zhao C, Zhao XY, Zhang HL, Shu HR, Hao YJ. MdCOP1 ubiquitin E3 ligases interact with MdMYB1 to regulate light-induced anthocyanin biosynthesis and red fruit coloration in apple. Plant Physiol. 2012;160:1011-22.

18. Peng T, Saito T, Honda C, Ban Y, Kondo S, Liu JH, Hatsuyama Y, Moriguchi T. Screening of UV-B-induced genes from apple peels by SSH: possible involvement of MdCOP1-mediated signaling cascade genes in anthocyanin accumulation. Physiol Plantarum. 2013;148:432-44.

19. Rizzini L, Favory JJ, Cloix C, Faggionato D, O'Hara A, Kaiserli E, Baumeister R, Schafer E, Nagy F, Jenkins GI, Ulm R. Perception of UV-B by the Arabidopsis UVR8 protein. Science. 2011;332:103-6.

20. Christie JM, Arvai AS, Baxter KJ, Heilmann M, Pratt AJ, O'Hara A, Kelly SM, Hothorn M, Smith BO. Plant UVR8 photoreceptor senses UV-B by tryptophan-mediated disruption of cross-dimer salt bridges. Science. 2012; 335:1492-6.

21. Wu D, Hu Q, Yan Z, Chen W, Yan C, Huang X, Zhang J, Yang P, Deng H, Wang J. Structural basis of ultraviolet-B perception by UVR8. Nature. 2012; 484:214-9.

22. Zuo Z, Liu H, Liu B, Liu X, Lin C. Blue light-dependent interaction of CRY2 with SPA1 regulates COP1 activity and floral initiation in Arabidopsis. Curr Biol. 2011;21:841-7.

23. Yanagawa Y, Sullivan JA, Komatsu S, Gusmaroli G, Suzuki G, Yin J, Ishibashi T, Saijo Y, Rubio V, Kimura S. Arabidopsis COP10 forms a complex with DDB1 and DET1 in vivo and enhances the activity of ubiquitin conjugating enzymes. Genes Dev. 2004;18:2172-81.

24. Gyula P, Schäfer E, Nagy F. Light perception and signalling in higher plants. Curr Opin Plant Biol. 2003;6:446-52.

25. Das PK, Shin DH, Choi SB, Park YI. Sugar-hormone cross-talk in anthocyanin biosynthesis. Mol Cells. 2012;34:501-7.

26. Shan X, Zhang Y, Peng W, Wang Z, Xie D. Molecular mechanism for jasmonate-induction of anthocyanin accumulation in Arabidopsis. J Exp Bot. 2009;60:3849-60.

27. Hoch WA, Singsaas EL, Mccown BH. Resorption protection. Anthocyanins facilitate nutrient recovery in autumn by shielding leaves from potentially damaging light levels. Plant Physiol. 2003;133:1296-305.

28. Gould KS, Mckelvie J, Markham KR. Do anthocyanins function as antioxidants in leaves? Imaging of $\mathrm{H}_{2} \mathrm{O}_{2}$ in red and green leaves after mechanical injury. Plant Cell Environ. 2002;25:1261-9.

29. Kytridis VP, Manetas Y. Mesophyll versus epidermal anthocyanins as potential in vivo antioxidants: evidence linking the putative antioxidant role to the proximity of oxy-radical source. J Exp Bot. 2006;57:2203-10.

30. Walz C, Juenger M, Schad M, Kehr J. Evidence for the presence and activity of a complete antioxidant defence system in mature sieve tubes. Plant J. 2002;31:189-97.

31. Rao MV, Paliyath G, Ormrod DP. Ultraviolet-B- and ozone-induced biochemical changes in antioxidant enzymes of Arabidopsis thaliana. Plant Physiol. 1996;110:125-36.

32. Hossain MA, Bhattacharjee S, Armin SM, Qian P, Xin W, Li HY, Burritt DJ, Fujita M, Tran LP. Hydrogen peroxide priming modulates abiotic oxidative stress tolerance: insights from ROS detoxification and scavenging. Front Plant Sci. 2015;6:420.

33. Chen C, Li H, Zhang D, Li P, Ma F. The role of anthocyanin in photoprotection and its relationship with the xanthophyll cycle and the antioxidant system in apple peel depends on the light conditions. Physiol Plantarum. 2013;149:354-66.

34. Wang L, Zhang X, Liu Y, Shi X, Wang Y, Zhang C, Zhao Z. The effect of fruit bagging on the color, phenolic compounds and expression of the anthocyanin biosynthetic and regulatory genes on the 'Granny Smith' apples. Eur Food Res Technol. 2013;237:875-85.

35. Ma C, Liang B, Chang B, Liu L, Yan J, Yang Y, Zhao Z. Transcriptome profiling reveals transcriptional regulation by DNA methyltransferase inhibitor 5-Aza-2'-deoxycytidine enhancing red pigmentation in bagged "Granny Smith" apples (Malus domestica). Int J Mol Sci. 2018;19:10.

36. Zhang XJ, Wang LX, Liu YL, Chen XX, Yang YZ, Zhao ZY. Differential gene expression analysis of 'Granny Smith' apple (Malus domestica Borkh.) during fruit skin coloration. S Afr J Bot. 2013;88:125-31.

37. Azuma A, Yakushiji H, Koshita Y, Kobayashi S. Flavonoid biosynthesis-related genes in grape skin are differentially regulated by temperature and light conditions. Planta. 2012;236:1067-80.

38. Bai SL, Sun YW, Qian MJ, Yang FX, Ni JB, Tao RY, Li L, Shu Q, Zhang D, Teng YW. Transcriptome analysis of bagging-treated red Chinese sand pear peels reveals light-responsive pathway functions in anthocyanin accumulation. Sci Rep-UK. 2017;7:63.

39. Wagner JR, Brunzelle JS, Forest KT, Vierstra RD. A light-sensing knot revealed by the structure of the chromophore-binding domain of phytochrome. Nature. 2005:438:325-31.

40. Jeong SW, Das PK, Jeoung SC, Song JY, Lee HK, Kim YK, Kim WJ, II Park Y, Yoo SD, Choi SB, Park YL. Ethylene suppression of sugar-induced anthocyanin pigmentation in Arabidopsis. Plant Physiol. 2010;154:1514-31.

41. Takada K, Ishimaru K, Minamisawa K, Kamada H, Ezura H. Expression of a mutated melon ethylene receptor gene cm-ETR1/H69A affects stamen development in Nicotiana tabacum. Plant Sci. 2005;169:935-42.

42. Chourey PS, Li QB, Kumar D. Sugar-hormone cross-talk in seed development: two redundant pathways of IAA biosynthesis are regulated differentially in the Invertase-deficient miniature1 ( $\mathrm{mn} 1$ ) seed mutant in maize. Mol Plant. 2010;3:1026-36.

43. Xie $X$, Song J, Wang Y, Sugar D. Ethylene synthesis, ripening capacity, and superficial scald inhibition in 1-MCP treated ' $d$ 'Anjou' pears are affected by storage temperature. Postharvest Biol Tec. 2014;97:1-10.

44. Jeong ST, Goto-Yamamoto N, Kobayashi S, Esaka M. Effects of plant hormones and shading on the accumulation of anthocyanins and the expression of anthocyanin biosynthetic genes in grape berry skins. Plant Sci. 2004;167:247-52.

45. Shen X, Zhao K, Liu L, Zhang K, Yuan H, Liao X, Wang Q, Guo X, Li F, $\mathrm{Li}$ T. A role for PacMYBA in ABA-regulated anthocyanin biosynthesis in red-colored sweet cherry cv. Hong Deng (Prunus avium L.). Plant Cell Physiol. 2014;55:862.

46. Sandhu AK, Gray DJ, Lu J, Gu L. Effects of exogenous abscisic acid on antioxidant capacities, anthocyanins, and flavonol contents of muscadine grape (Vitis rotundifolia) skins. Food Chem. 2011;126:982-8.

47. Li D, Luo Z, Mou W, Wang Y, Ying T, Mao L. ABA and UV-C effects on quality, antioxidant capacity and anthocyanin contents of strawberry fruit (Fragaria ananassa Duch.). Postharvest Biol Tec. 2014;90:56-62.

48. Loreti E, Povero G, Novi G, Solfanelli C, Alpi A, Perata P. Gibberellins, jasmonate and abscisic acid modulate the sucrose-induced expression of anthocyanin biosynthetic genes in Arabidopsis. New Phytol. 2008;179: 1004-16.

49. Deikman J, Hammer PE. Induction of anthocyanin accumulation by cytokinins in Arabidopsis thaliana. Plant Physiol. 1995;108:47-57.

50. Hwang I, Sheen J. Two-component circuitry in Arabidopsis cytokinin signal transduction. Nature. 2001;413:383-9.

51. Qi T, Song S, Ren Q, Wu D, Huang H, Chen Y, Fan M, Peng W, Ren C, Xie D. The Jasmonate-ZIM-domain proteins interact with the WD-repeat/bHLH/ MYB complexes to regulate Jasmonate-mediated anthocyanin accumulation and trichome initiation in Arabidopsis thaliana. Plant Cell. 2011;23:1795-814.

52. Miller G, Suzuki N, Rizhsky L, Hegie A, Koussevitzky S, Mittler R. Double mutants deficient in cytosolic and thylakoid ascorbate peroxidase reveal a complex mode of interaction between reactive oxygen species, plant development, and response to abiotic stresses. Plant Physiol. 2007;144: 1777-85.

53. Willekens H, Langebartels C, Tire C, Van Montagu M, Inze D, Van Camp W. Differential expression of catalase genes in Nicotiana plumbaginifolia (L.). P Natl Acad Sci USA. 1994;91:10450-4.

54. Skadsen RW, Schulze-Lefert P, Herbst JM. Molecular cloning, characterization and expression analysis of two catalase isozyme genes in barley. Plant Mol Biol. 1995;29:1005-14.

55. Sarowar S, Kim EN, Kim YJ, Ok SH, Kim KD, Hwang BK, Shin JS. Overexpression of a pepper ascorbate peroxidase-like 1 gene in tobacco plants enhances tolerance to oxidative stress and pathogens. Plant Sci. 2005;169:55-63.

56. Gill SS, Tuteja N. Reactive oxygen species and antioxidant machinery in abiotic stress tolerance in crop plants. Plant Physiol Bioch. 2010;48:909-30

57. Eltayeb AE, Kawano N, Badawi GH, Kaminaka H, Sanekata T, Shibahara T, Inanaga S, Tanaka K. Overexpression of monodehydroascorbate reductase in transgenic tobacco confers enhanced tolerance to ozone, salt and polyethylene glycol stresses. Planta. 2007;225:1255-64.

58. Yoshimura K, Miyao K, Gaber A, Takeda T, Kanaboshi H, Miyasaka H, Shigeoka S. Enhancement of stress tolerance in transgenic tobacco plants overexpressing chlamydomonas glutathione peroxidase in chloroplasts or cytosol. Plant J. 2004;37:21-33.

59. Gaber A, Yoshimura K, Yamamoto T, Yabuta Y, Takeda T, Miyasaka H, Nakano Y, Shigeoka S. Glutathione peroxidase-like protein of Synechocystis 
PCC 6803 confers tolerance to oxidative and environmental stresses in transgenic Arabidopsis. Physiol Plantarum. 2006;128:251-62.

60. Wang Z, Du H, Zhai R, Song L, Ma F, Xu L. Transcriptome analysis reveals candidate genes related to color fading of 'Red Bartlett' (Pyrus communis L.). Front Plant Sci. 2017;8:455

61. Murashige T, Skoog F. A revised medium for rapid growth and bio assays with tobacco tissue cultures. Physiol Plantarum. 1962;15:473-97.

62. Lichtenthaler HK, Wellburn AR. Determinations of total carotenoids and chlorophylls $\mathrm{a}$ and $\mathrm{b}$ of leaf extracts in different solvents. Biochem Soc Trans. 1983;11:591.

63. Mortazavi A, Williams BA, McCue K, Schaeffer L, Wold B. Mapping and quantifying mammalian transcriptomes by RNA-Seq. Nat Methods. 2008;5:621.

64. Velasco R, Zharkikh A, Affourtit J, Dhingra A, Cestaro A, Kalyanaraman A, Fontana P, Bhatnagar SK, Troggio M, Pruss D. The genome of the domesticated apple (MalusX domestica Borkh.). Nat Genet. 2010;42:833.

65. Li R, Yu C, Li Y, Lam T-W, Yiu S-M, Kristiansen K, Wang J. SOAP2: an improved ultrafast tool for short read alignment. Bioinformatics. 2009;25: $1966-7$.

66. Trapnell C, Roberts A, Goff L, Pertea G, Kim D, Kelley DR, Pimentel H, Salzberg SL, Rinn JL, Pachter L. Differential gene and transcript expression analysis of RNA-seq experiments with TopHat and cufflinks. Nat Protoc. 2012;7:562.

67. Kim D, Pertea G, Trapnell C, Pimentel H, Kelley R, Salzberg SL. TopHat2 accurate alignment of transcriptomes in the presence of insertions, deletions and gene fusions. Genome Biol. 2013;14:1-13.

68. Benjamini Y, Hochberg Y. Controlling the false discovery rate: a practical and powerful approach to multiple testing. J R Stat Soc B. 1995;57:289-300.

69. Huang DW, Sherman BT, Lempicki RA. Systematic and integrative analysis of large gene lists using DAVID bioinformatics resources. Nat Protoc. 2008;4:44.

70. Kanehisa M, Araki M, Goto S, Hattori M, Hirakawa M, Itoh M, Katayama T, Kawashima S, Okuda S, Tokimatsu T. KEGG for linking genomes to life and the environment. Nucleic Acids Res. 2007;36:480-4.

71. Zhang B, Horvath S. A general framework for weighted gene co-expression network analysis. Stat Appl Genet Mol. 2005;4:1-45.

72. Shannon P, Markiel A, Ozier O, Baliga NS, Wang JT, Ramage D, Amin N, Schwikowski B, Ideker T. Cytoscape: a software environment for integrated models of biomolecular interaction networks. Genome Res. 2003;13:2498-504.

73. Saldanha AJ. Java Treeview-extensible visualization of microarray data. Bioinformatics. 2004;20:3246-8.

74. Wurch T, Lestienne F, Pauwels P. A modified overlap extension PCR method to create chimeric genes in the absence of restriction enzymes. Biotechnol Tech. 1998;12:653-7.

75. Xu M, Zhang X, Zhang L, Luo Y, Fan Y, Wang L. Functional analysis of BnMAR element in transgenic tobacco plants. Mol Biol Rep. 2011;38:3285-91.

76. Drumm-Herrel $\mathrm{H}$, Mohr H. Effect of BLUE/UV light on anthocyanin synthesis in tomato seedings in the absence of bulk carotenoids. Photochem Photobiol. 2010;36:229-33.

77. Jefferson RA, Kavanagh TA, Bevan MW. GUS fusions: beta-glucuronidase as a sensitive and versatile gene fusion marker in higher plants. EMBO J. 1987;6: 3901-7.

78. Bradford MM. A rapid and sensitive method for the quantitation of microgram quantities of protein utilizing the principle of protein-dye binding. Anal Biochem. 1976;72:248-54.

79. Livak K, Schmittgen TD. Analysis of relative gene expression data using real-time quantitative PCR and the $2^{-\Delta \Delta C T}$ method. Methods. 2001;25:402-8.

Ready to submit your research? Choose BMC and benefit from:

- fast, convenient online submission

- thorough peer review by experienced researchers in your field

- rapid publication on acceptance

- support for research data, including large and complex data types

- gold Open Access which fosters wider collaboration and increased citations

- maximum visibility for your research: over $100 \mathrm{M}$ website views per year

At BMC, research is always in progress.

Learn more biomedcentral.com/submissions 